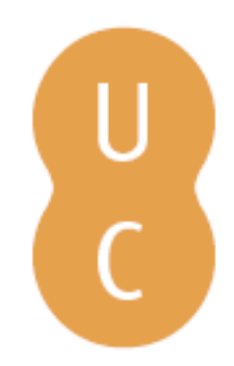

\title{
nommalina
}

\section{Amatus Lusitanus e Didaco Pirro: due ebrei portoghesi e cerchia umanistica di Dubrovnik}

\author{
Autor(es): $\quad$ Lupis, Vinicije B.
}

Publicado por: UA Editora - Universidade de Aveiro; Imprensa da Universidade de

URL persistente:

URI:http://hdl.handle.net/10316.2/35707

DOI:

DOI:http://dx.doi.org/10.14195/978-989-26-0941-6_21

Accessed : $\quad$ 26-Apr-2023 08:01:36

A navegação consulta e descarregamento dos títulos inseridos nas Bibliotecas Digitais UC Digitalis, UC Pombalina e UC Impactum, pressupõem a aceitação plena e sem reservas dos Termos e Condições de Uso destas Bibliotecas Digitais, disponíveis em https://digitalis.uc.pt/pt-pt/termos.

Conforme exposto nos referidos Termos e Condições de Uso, o descarregamento de títulos de acesso restrito requer uma licença válida de autorização devendo o utilizador aceder ao(s) documento(s) a partir de um endereço de IP da instituição detentora da supramencionada licença.

Ao utilizador é apenas permitido o descarregamento para uso pessoal, pelo que o emprego do(s) título(s) descarregado(s) para outro fim, designadamente comercial, carece de autorização do respetivo autor ou editor da obra.

Na medida em que todas as obras da UC Digitalis se encontram protegidas pelo Código do Direito de Autor e Direitos Conexos e demais legislação aplicável, toda a cópia, parcial ou total, deste documento, nos casos em que é legalmente admitida, deverá conter ou fazer-se acompanhar por este aviso.

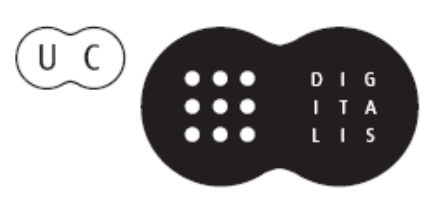




\section{HUMANISMO E CIÊNCIA Antiguidade e Renascimento}

António Manuel Lopes Andrade

Carlos de Miguel Mora

João Manuel Nunes Torrão
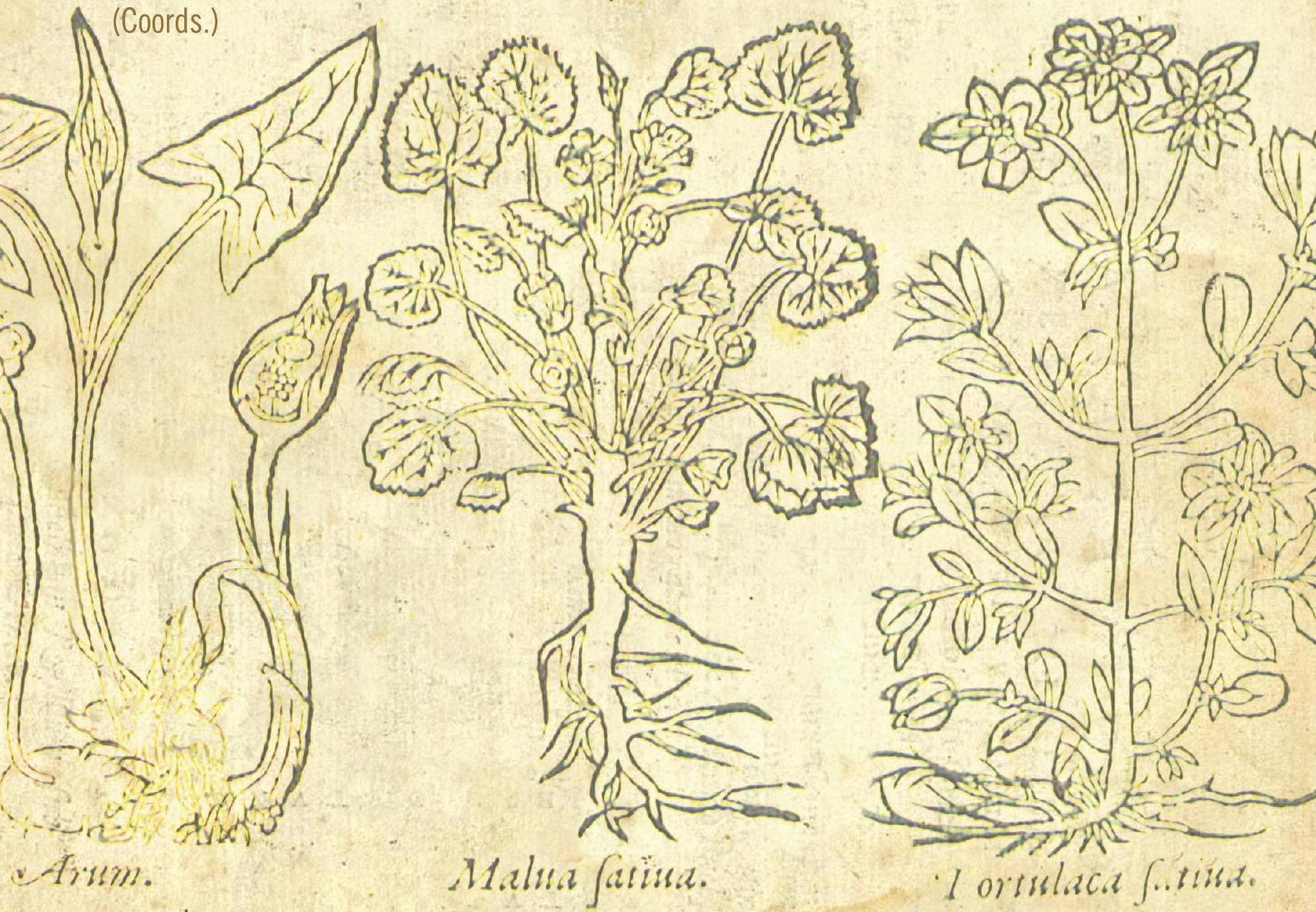

Aveiro I Coimbra I São Paulo 2015

UA Editora - Universidade de Aveiro I Imprensa da Universidade de Coimbra I Annablume 
Este volume resulta de várias iniciativas desenvolvidas no âmbito do projecto de I\&D "Dioscórides e o Humanismo Português: os Comentários de Amato Lusitano" (http://amatolusitano.web.ua.pt), recoIhendo contribuições de mais de duas dezenas de colaboradores, tanto de membros da equipa como de outros investigadores nacionais e estrangeiros. Entre os eventos que estiveram na origem deste livro destacam-se as três edições do Ciclo de Conferências promovido pelo projecto, realizadas entre 2010 e 2013, e sobretudo o Colóquio Internacional "Dioscórides e o Humanismo Português: os Comentários de Amato Lusitano", que decorreu no Departamento de Línguas e Culturas da Universidade de Aveiro, nos dias 21 e 22 de Novembro de 2013.

0 objectivo principal do projecto é a edição e tradução para português dos dois livros que Amato Lusitano dedicou ao comentário do tratado grego De materia medica de Dioscórides, ou seja, o Index Dioscoridis (Antuérpia, 1536) e as In Dioscoridlis Anazarbei de medica materia libros quinque... enarrationes (Veneza, 1553), estando contemplada, também, a tradução de mais duas obras directamente correlacionadas com os livros do médico português: a montante, a do próprio tratado grego de Dioscórides; a jusante, a do livro intitulado Apologia adversus Amathum Lusitanum (Veneza, 1558) de Pietro Andrea Mattioli.

OBRA PUBLICADA COM A COORDENAÇÃO

CIENTÍFICA DE:

Centro de Línguas, Literaturas e Culturas da Universidade de Aveiro

Centro de Estudos Clássicos e Humanísticos da Universidade de Coimbra

Cátedra de Estudos Sefarditas "Alberto

Benveniste" da Faculdade de Letras da Universidade de Lisboa 


\section{HUMANISMO E CIÊNCIA}

\section{Antiguidade e Renascimento}

ANTÓNIO MANUEL LOPES ANDRADE

CARLOS DE MIGUEL MORA

JOÃO MANUEL NUNES TORRÃO

(COORDS.)

AVEIRO • COIMBRA • SÃO PAULO

2015

UA EDITORA • UNIVERSIDADE DE AVEIRO

IMPRENSA DA UNIVERSIDADE DE COIMBRA

ANNABLUME 


\section{HUMANISMO E CIÊNCIA: Antiguidade e Renascimento}

\author{
EDIÇÃO

UA EDITORA • UNIVERSIDADE DE AVEIRO
IMPRENSA DA UNIVERSIDADE DE COIMBRA
ANNABLUME

ORGANIZAÇÃO E COORDENAÇÃO EDITORIAL ANTÓNIO MANUEL LOPES ANDRADE

CARLOS DE MIGUEL MORA

JOÃO MANUEL NUNES TORRÃO

\author{
DESIGN DA CAPA \\ MEIOKILO DESIGN STUDIO
}

DESIGN

CARLOS COSTA

IMPRESSÃO E ACABAMENTO

SERSILITO • MAIA

ISBN

UA • 978-972-789-434-5

IUC • 978-989-26-0940-9

\section{ISBN DIGITAL}

UA • 978-972-789-435-2

IUC • 978-989-26-0941-6

DOI

http://dx.doi.org/10.14195/ 978-989-26-0941-6

DEPÓSITO LEGAL 368241/13

TIRAGEM 500 Exemplares

(C) 2015

UA EDITORA • UNIVERSIDADE DE AVEIRO IMPRENSA DA UNIVERSIDADE DE COIMBRA

ANNABLUME

\section{COMISSÃO CIENTÍFICA}

António Manuel Lopes Andrade

Carlos de Miguel Mora

Delfim Ferreira Leão

Henrique Leitão

João Manuel Nunes Torrão

Maria de Fátima Reis

Maria do Céu Zambujo Fialho

Miguel Ángel González Manjarrés

\section{TEXTOS}

Adelino Cardoso

Ana Leonor Pereira

Ana Margarida Borges

António Guimarães Pinto

António Maria Martins Melo

Bernardo Mota

Carlos A. Martins de Jesus

Carlos de Miguel Mora

Cristina Santos Pinheiro

Donald Beecher

Emília Oliveira

Isabel Malaquias

James W. Nelson Novoa

Joana Mestre Costa

João Manuel Nunes Torrão

João Rui Pita

Jorge Paiva

José Sílvio Moreira Fernandes

Maria de Fátima Silva

Miguel Ángel González Manjarrés

Rui Manuel Loureiro

Telmo Corujo dos Reis

Teresa Nobre de Carvalho

Vinicije B. Lupis

Virgínia Soares Pereira 


\section{HUMANISMO E CIÊNCIA}

\section{Antiguidade e Renascimento}

ANTÓNIO MANUEL LOPES ANDRADE

CARLOS DE MIGUEL MORA

JOÃO MANUEL NUNES TORRÃO

(COORDS.)

AVEIRO • COIMBRA • SÃO PAULO

2015

UA EDITORA • UNIVERSIDADE DE AVEIRO

IMPRENSA DA UNIVERSIDADE DE COIMBRA

ANNABLUME 
OBRA PUBLICADA

COM A COORDENAÇÃO

CIENTÍFICA DE:

CENTRO DE LÍNGUAS,

LITERATURAS E CULTURAS DA

UNIVERSIDADE DE AVEIRO

CENTRO DE ESTUDOS

CLÁSSICOS E HUMANÍSTICOS DA

UNIVERSIDADE DE COIMBRA

CÁTEDRA DE ESTUDOS SEFARDITAS

"ALBERTO BENVENISTE"

DA FACULDADE DE LETRAS DA

UNIVERSIDADE DE LISBOA 


\section{SUMÁRIO}

PREFÁCIO

1.1 "Teofrasto, Tratado das plantas. No alvor de uma nova ciência" 13

Maria de Fátima Silva

1.2 "Francisco de Melo e os fragmentos de teoria óptica de Pierre Brissot" 21 Bernardo Mota

1.3 "Algumas reflexões sobre as pedras preciosas nos Colóquios dos simples de Garcia de Orta" 37 Rui Manuel Loureiro

1.4 "Estratégias, patronos e favores em Colóquios dos Simples de Garcia de Orta" 63 Teresa Nobre de Carvalho

1.5 "As plantas na obra poética de Camões (épica e lírica)" 95 Jorge Paiva

1.6 "Nicolás Monardes, John Frampton and the Medical Wonders of the New World" .141 Donald Beecher

1.7 "Literatura e Medicina: alguns textos de Justo Lípsio e de dois doutores Luís Nunes" 161 António Guimarães Pinto

1.8 "Ontologias e idiossincrasias dos Amantes, à luz da Archipathologia de Filipe Montalto" ...... 211 Joana Mestre Costa \& Adelino Cardoso

1.9 "Gabriel da Fonseca. A New Christian doctor in Bernini's Rome". .227 James W. Nelson Novoa 


\section{2) DIOSCÓRIDES E O HUMANISMO PORTUGUÊS: OS COMENTÁRIOS DE AMATO LUSITANO}

2.1 "Léxico científico português nos Comentários de Amato: antecedentes e receção"

Ana Margarida Borges

2.2 "Usos medicinais das plantas, em Amato Lusitano: o bálsamo"

António Maria Martins Melo

2.3 "Amato Lusitano e a importância da ilustração botânica no século xVI.

Em torno das edições lionesas das Enarrationes (1558)"

Carlos A. Martins de Jesus

2.4 "Sobre la identificación entre ébano y guayaco en una entrada

del Index Dioscoridis de Amato Lusitano".

Carlos de Miguel Mora

2.5 "Os partos distócicos em Amato Lusitano e em Rodrigo de Castro:

fontes, doutrinas e terapias greco-romanas"

\section{Cristina Santos Pinheiro}

2.6 "Do carvalho ao castanheiro: usos e propriedades medicinais

de fagáceas nas Enarrationes de Amato Lusitano".

Emília Oliveira

2.7 "O mundo mineral nos Comentários a Dioscórides de Amato Lusitano".

Isabel Malaquias \& Virgínia Soares Pereira

2.8 "Alguns comentários de Amato: entre a estranheza e a realidade"

João Manuel Nunes Torrão

2.9 "Caracterização e usos terapêuticos de produtos de origem marinha

nos Comentários de Amato Lusitano a Dioscórides"

José Sílvio Moreira Fernandes

2.10 "La mandrágora de Amato Lusitano: edición, traducción y anotación"

Miguel Ángel González Manjarrés

2.11 "O vinho e os vinhos - usos e virtudes de um dom dos deuses

nas Enarrationes de Amato Lusitano"

Telmo Corujo dos Reis

2.12 "Amatus Lusitanus e Didaco Pirro: due ebrei portoghesi

e cerchia umanistica di Dubrovnik" 481

Vinicije B. Lupis

2.13 "Estudos contemporâneos sobre Amato Lusitano". 513

João Rui Pita \& Ana Leonor Pereira 


\section{Amatus Lusitanus e Didaco Pirro: due ebrei portoghesi e cerchia umanistica di Dubrovnik}

VINICIJE B. LUPIS 1

\section{RIASSUNTO:}

Amatus Lusitanus, medico e medico scrittore, e Didacus Pyrrhus Lusitanus (Diogo Pires, Didaco Pirro o Didak Pir), due personalità interessanti del periodo umanistico, nel xVI secolo hanno collegato la Croazia e il Portogallo. Allora Dubrovnik era un centro culturale ed economico estremamente importante di questa zona europea. Nella primavera dell'anno 1556 Amatus arrivò nella città di Dubrovnik, dove rimase per due anni. Curava molti patrizi e plebei di Dubrovnik e aveva pazienti dall'Impero Ottomano. L'opera più importante di Amatus e Curationum Medicinalium Centuriae, cioè le 700 storie più interessanti accadutegli nell'ambito del suo mestiere e seguite da personali interpretazioni (scholia). Per noi, la storia più interessante è la sesta centuria (Ragusina), pubblicata a Lyon nel 1564, contenente 100 casi di pratica di Amatus a Dubrovnik, fornisce un quadro straordinario sulla vita culturale e sanitaria della Dubrovnik rinascimentale. Tra i pazienti di Amatus a Dubrovnik vi era anche Sabo Bobaljević Mišetić Glušac (Dubrovnik, 1529/1530 - Ragusa, 1585), un patrizio e poeta malato di sifilide. Tra le sue opere il Canzoniere italiano, pubblicato postumo a Venezia nel 1589, e la rielaborazione in croato dell'Amor fuggitivo di Tasso. Tramite un lungo soggiorno e le opere letterarie, Didak Pir lasciò un segno incancellabile nella città ragusea. Didak Pir ha ringraziato la sua nuova patria - Dubrovnik - nelle lettere scritte in esametro a Paolo Manuzio, figlio del famoso editore Aldo (Ad Paulum, 1563). Con lo stesso spirito stese la "canzone delle famiglie importanti di Dubrovnik d'oggi" (De illustribus familiis quae hodie Rhacusae exstant, 1582), per la quale fu insignito dal Senato di Dubrovnik.

\section{PAROLE CHIAVE:}

Amatus Lusitanus; Didaco Pirro; Dubrovnik; Toma Budislavić; Medicina

1 Istituto di Scienze Sociali "Ivo Pilar" - Centro regionale in Dubrovnik - Croatia: vinicije.lupis@pilar.hr. 


\begin{abstract}
:
Amatus Lusitanus, physician and medical writer, and Didacus Pyrrhus Lusitanus (Didaco Pirro or Didak Pir) were two interesting personalities of the humanistic period. They have linked Croatia and Portugal in the sixteenth century. Dubrovnik was at the time extremely important cultural and economic center of this part of Europe. In spring of 1556 Amatus arrived in the city of Dubrovnik, where he remained for two years. He looked after many patricians and plebeians of Dubrovnik and had patients from the Ottoman Empire. The most important work of Amatus was Curationum Medicinalium Centuriae, with 700 most interesting stories of his practice with interpretations (scholia). For us the most interesting part of this book is sixth centurion (Ragusina), with 100 instances of the practice of Amatus in Dubrovnik because it gives us an excellent picture of the cultural life and health in Dubrovnik Renaissance. Among other patients of Amatus in Dubrovnik was also the patrician and poet who suffered from syphilis - Sabo Bobaljević Mišetić Glušac (Dubrovnik, 1529/1530 - Dubrovnik, 1585). His Italian Canzoniere was published posthumously in Venice in 1589. He also reworked Tasso's Amor fuggitivo in Croatian. Didak Pir was another important person of Portuguese-Croatian humanistic relations. At his new home - Dubrovnik, Didacus Pyrrhus Lusitanus thanked in his letters (written in hexameter verse) to Paulo Manutius. In the same spirit he wrote the song about most important families of Dubrovnik (De illustribus familiis quae hodie Rhacusae exstant, 1582), for which he was awarded by the Dubrovnik Senate.
\end{abstract}

\title{
KEYWORDS:
}

Amatus Lusitanus; Didacus Pyrrhus Lusitanus; Dubrovnik; Toma Budislavić; Medicine 
La Città-Stato di Dubrovnik (in italiano Ragusa) era estesa circa $1.500 \mathrm{Km}^{2}$ nel periodo della sua massima espansione, con una popolazione oscillante tra 25.000 e 75.000 abitanti fra XVI e XviII secolo, dei quali mediamente non più di 6-7.000 nella capitale. ${ }^{2}$ Forte di una flotta mercantile di circa 200 navi tra grandi e piccole, per un totale di 35.000 carri di tonnellaggio attorno al 1570 - alla pari di quelle di Venezia, Genova, Marsiglia, Napoli e Sicilia, come ha rilevato Braudel $^{3}$ - il commercio marittimo raguseo nel corso del'500 non solo si dispiegava nel Mediterraneo orientale e occidentale, ma altresì nell'Atlantico, essendo attiva per gran parte di questo secolo la linea Dubrovnik — Londra. La seconda città dello Stato è Stagno, la terza è Ragusa Vecchia o Cavtat. Già nei primi decenni del xvi secolo Ragusa - consapevole della propria fragilità, stretta com'è tra terre veneziane e ottomane — attende a migliorare la difesa, sviluppare la flotta, potenziare il porto, stringere più larghe e sicure relazioni internazionali. Dubrovnik, città-stato da 1358, non potrebbe mai contare sulle proprie forze per proteggersi da violenze turche e veneziane. È alle arti della diplomazia che essa deve continuamente ricorrere per garantirsi neutralità indispensabile per mantenere relazioni commerciali con tutte le potenze. Così paga un tributo annuale alla Sublime Porta (dopo averlo a lungo pagato al regno Croato-Ungaro), fa continue professioni di ortodossia cattolica al papa, affittava navi ed equipaggio alla Spagna, cerca di imbonire Venezia, passandole qualche informazione sulle cose dei Turchi, mantiene cordiali relazioni con Ancona e con gli stati di Urbino e Ferrara, dispone di consoli nell'Italia spagnola e intrattiene ottimi rapporti con la Francia. Un miracolo di abilità diplomatica nel convulso mondo mediterraneo del xvi secolo. E se è vero che la comunità di Ragusa viveva perennemente intimorita, è anche vero che, alla fine (1806) nessun esercito nemico abbia mai alzato uno stendardo sulle propie mura. Una struttura politica rigidamente aristocratica, con il maggior consiglio serrato intorno 1320, una stretta commissione di affari pubblici e privati, controllata da antiche famiglie, un'indiscutibile correttezza mercantile unita a grande spirito di intraprendenza, una non comune pazienza di fronte alla pretese di Venezia, della Spagna e di Constantinopoli, sono tra gli elementi caratteristici di questo Stato (chiamato dispettivamente "sette bandiere"), che, in fondo, la gente poco affidabile a Venezia, poco tollerante con l'eccessiva indipendenza di rivali commerciali “in golfo”. Ragusa, Ancona, Venezia: un triangolo di rivalità e complementarità ad un tempo, che ha visto, momenti di rottura profonda, ma ha anche registrato fasi di collaborazione. E basterebbe pensare alla vicenda di molte famiglie di Dubrovnik come quelle di Kabužić, Gučetić, Sorkočević, Zuzorić, Gundulić, Bunić, Pucić, Restić ecc., detentrici contemporaneamente di imprese commerciali a

2 Zdravko ŠUndRICA, "Popis stanovništva Dubrovačke republike iz $1673-74$ godine", Arhivski vjesnik 2 (1959), pp. 419-456; F. W. CARTER, Dubrovnik (Ragusa). A Classic City-State. London-New York, Seminar Press, 1972, pp. 124.

3 Ferdinand Braudel, Civiltà e Imperi del Mediterraneo nell'età di Filippo II. Torino, Einaudi, 1982, vol. I., pp. 477. 
Belgrado, Venezia, Ancona, Londra e Dubrovnik, ognuna diretta da un componente familiare o da un socio. E i traffici continuano con certà regolarità anche in tempo di guerra. Ancona è la porta orientale di Roma e Firenze, ma Dubrovnik è uno dei maggiori accessi all'Impero turco per i generi ponentini. ${ }^{4}$ Scrive Lodovico Beccadelli, arcivescovo di Dubrovnik (1555-1564), al governatore di Ancona: "parmi ad un modo ci siamo tutti in una città medesima, che così reputo Ancona et Ragusa per il frequente commercio ch'hanno insieme, et li spessi passaggi dall'una all'altra". ${ }^{5}$ Nel xvi secolo Dubrovnik è la porta dell'Europa per l'Impero Ottomano, una città commerciale estremamente sensibile a qualsiasi tipo di coinvolgimento in conflitti armati, e ha sviluppato una particolare cura per l'arrivo di stranieri sul proprio territorio.

\section{AMATUS LUSITANUS - AMATO LUSITANO}

Amatus Lusitanus, medico e noto autore di testi medicinali (Castelo Branco, Portogallo, 1511 - Salonicco, 21 gennaio 1568) era marrano, cioè, ebreo fattosi cristiano. Compiuti gli studi di medicina a Salamanca, esercitava la professione medica nelle diverse città portoghesi e spagnole. Dietro l'invito di assumere la carica di medico statale a Dubrovnik, venne nel 1547 ad Ancona dove negoziava le condizioni di servizio con il Senato di Ragusa/Dubrovnik. Le trattative furono lunghe per cui, nel frattempo, esercitava la professione ad Ancona e Roma, dove per un breve tempo fu il medico del papa Giulio III (1550). Poi fuggì a Pesaro a causa della persecuzione degli ebrei. ${ }^{6}$

Nella primavera del 1556 venne a Dubrovnik e vi restò, secondo lo storico Lavoslav Glesinger, due anni. ${ }^{7}$ La tesi di Glesinger sembra essere ben fondata. Con tale datazione è d'accordo anche dalla storica Vesna Miović-Perić. La tesi è corroborata anche mediante la comparazione di fonti archivistiche e con l'età dei suoi pazienti menzionati nel suo libro. ${ }^{8}$

4 Sergio AnSELMI, "Motivazioni economiche della neutralità di Ragusa nel cinquecento" in Antonio Di Vittorio, Sergio Anselmi, Paola Pierucci, Ragusa (Dubrovnik) una repubblica adriatica. Saggi di storia economica e finanziaria. Bologna, Cisalpino, 1994, pp. 25-34.

5 Josip Torbarina, "Fragmenti iz neizdanih pisama Lodovika Beccadellija 1555-1564", Dubrovačka, mjesečna ilustrovana revijal (1929), pp. 9-10.

6 António Manuel Lopes ANDRADE; Hugo Miguel CRESPo, "Os inventários dos bens de Amato Lusitano, Francisco Barbosa e Joseph Molcho, em Ancona, na fuga à Inquisição (1555)", Ágora. Estudos Clássicos em Debate 14.1 (2012), pp. 45-90.

7 Lavoslav GLESINGER, Amatus Lusitanus i njegov lječnički rad u Dubrovniku (1556-1558). Zagreb-Beograd, Biblioteka Jevrejskog narodnog kalendara, 1940; idem, "Amatus Lusitanus i njegov liječnički rad u Dubrovniku (1556-1558)", Omanut 3-4 (1940), pp. 61-62; idem,"Dubrovački lječnik - prvi borac protiv progona vještica", Zaštita zdravlja 3 (1948), pp. 106-107.

8 Vesna Mıović, Židovski geto u Dubrovačkoj Republici (1546-1808). Dubrovnik-Zagreb, HAZU, Zavod za Povijesne Znanosti u Dubrovniku, 2005, p. 24. 
La venuta del Lusitano a Dubrovnik coincide con il ritiro di Carlo V d'Absburgo nel monastero Yuste e l'abdicazione a favore del figlio Filippo II e fratello Ferdinando. Lo storico Bariša Krekić sostiene che Amatus arrivò a Dubrovnik verso la fine del 1557. Dello stesso parere è anche un altro storico, Jorjo Tadić. ${ }^{9}$ Lo storico di Dubrovnik Francesco Maria Appendini sosteneva che Amatus si trattenne a Dubrovnik dal 1551 al 1558. Il governo voleva trattenerlo ancora, ma lui declinò l'invito e partì per Salonicco. ${ }^{10}$ Šime Ljubić conferma la tesi dell'Appendini circa il soggiorno di Amatus Lusitanus a Dubrovnik tra il 1551 e il $1558 .{ }^{11}$ Una notizia del 1557 ci dice che a Dubrovnik si trovasse un medico ebreo interdetto dal governo per l'esercizio ulteriore del suo mestiere. ${ }^{12}$ L'arrivo di Amatus ebbe luogo in un tempo di grande prosperità economica di Dubrovnik, e i marrani qui venuti non ebbero un ruolo sostanziale per la città di Dubrovnik, per cui i loro interessi erano complementari con quelli ragusei. ${ }^{13}$ Amatus curava molti cittadini di Ragusa, sia nobili sia popolani, nonché pazienti venuti dal territori turci, e si presenta perfino come esperto dinanzi al tribunale, ma non riesce a ottenere un posto nell'esercizio statale a causa degli intrighi dei medici ragusei. All'inizio del mese di febbraio del 1558 fu aggredito di notte il chirurgo Gianbattista Vanucci, il quale non riconobbe l'aggressore. Cinque anni prima dell'incidente il patrizio raguseo Giugno Bobaljević (Bobali), fratello di Savino, paziente dell'Amatus Lusitanus, aggredì il medico Giacomo Paccino. Il 23 luglio 1550 il detto Paccino aveva a sua volta aggredito il medico

9 Bariša KREKIĆ, "The role of the Jews in Dubrovnik (thirteenth-sixteenth centuries)", Viator (Medieval and Renaissance Studies) 4 (1973), p. 269; Jorjo TADIĆ, Jevreji u Dubrovniku do polovine XVII. Stoljeća. Sarajevo, Izdala La Benevolencia, 1937, p. 284.

10 Francesco Maria APPEndinı, Notizie istorico-critiche sulle antichità, storia e letteratura de'Ragusei, vol. II. Ragusa, dalle stampe di Antonio Martecchini, 1803, p. 324.

11 Šime LJUBIĆ, Dizionario biografico degli uomini illustri della Dalmazia. Beč, 1856, p. 190: "LUSITANO Amato, portoghese, venne in Ragusa nel 1551 ad esercitarvi l'arte salutare, e nel 1558 si ridusse in Salonichio, ove si fece ebreo e morì. Nella sua opera medica: Centuriae VII. curationum medicinalium dedica la sesta centuria all'analisi de'morbi endemici di Ragusa e suo teritorio".

12 J. TADIč, Jevreji u..., op. cit., p. 249; Državni arhiv u Dubrovniku (DAD), Acta Minoris Consilii, V. (Con. Minus), XLIV, fol. 206.

13 B. KREKIČ, "The role...", op. cit., pp. 270-271; T. MORTIDJIJA, "Židovi u starom Dubrovniku", Hrvatska straža 6 (1934), pp. 221-225; Šalom FreiberGer, "Povijest Židova u Dubrovniku do izgona god. 1515", Omanut 1 (1936), pp. 30-37; David GINSBERG, "Mletački rabin Leo de Modena i dubrovački pjesnik Vinko Komnenović", Omanut 10 (1940), pp. 156-161; Zbornik Židovskog muzeja, vol. 1., contiene "Studije i gradja o Jevrejima u Dubrovniku", Beograd, 1971: Radovan SAMARDžıč, "Dubrovački Jevreji u trgovini xVI i xVII veka", (pp. 21-39), T. Popovıč, "Dubrovnik i Ankona u jevrejskoj trgovini xvı veka", (pp. 41-53); Bogumil HrabaK, "Jevreji u Albaniji od kraja XIII do kraja XVII veka i njihove veze s Dubrovnikom", (pp. 55-97); Ladislav MONSTER, "Proces zbog "ritualnog ubistva" u Dubrovniku 1502 godine i tragična smrt lekara Moše Maralija", (pp. 99-112); Miroslav PANTIČ, "Jevreji u dubrovačkoj književnosti", (pp. 211-238); D. Nevenič-Grabovac, "Iz poezije Didaka Pira", (pp. 253-290); Lavoslav Glesinger, "Dubrovački lječnik Amatus Lusitanus", (pp. 291-312); Mirjana Gušıč, "Neki primjerci tekstila u zbirci sinagoge jevrejske općine u Dubrovniku", (pp. 325-336); Z. Efron, "Jevrejski nadgrobni spomenici u Dubrovniku", (pp. 337-340). 
ebreo Isacco, durante un consiglio medico nel monastero dei frati minori di Dubrovnik. ${ }^{14}$ Fu aperta un'istruttoria, che però non sortì alcun risultato. In occasione dell'incidente con Vanucci l'istruttoria fu condotta in Senato dal 11 fino al 25 febbraio, ma senza giungere a un esito finale. Un mese dopo il chirurgo Vanucci fece istanza di rimborso per un periodo di tre mesi di vacanze non retribuito a causa degli affari aperti in Italia. ${ }^{15}$ Va notato che egli aveva contatti di affari con parecchi patrizi e cittadini pazienti del Lusitano, tra i quali Sjepan Gučetić (Gozze), figlio di Antonio, Bernard Zuzorić (Zuzzeri), Pavao di Luka Sorkočević (Sorgo), Vlaho di Nikola Držić (Darsa). ${ }^{16}$ Durante l'anno 1556 Vanucci ebbe contatti d'affari con Dominik Kladorubović — compagno del Šimun di Miho Zlatarić. ${ }^{17}$ In questo periodo Amatus esercitava la professione medica, ma certi cittadini ragusei si ribellarono contro i medici ebrei. Per questo il 23 marzo 1558 il governo raguseo prese una decisione in tal senso e questa due giorni dopo fu prontamente comunicata al Lusitano. ${ }^{18}$ Egli però continuava l'esercizio professionale senza procacciarsi il permesso dell'arcivescovo. Quando il medico Vanucci lasciò Dubrovnik per le vacanze trimestrali, il 28 aprile 1558 Amatus richiese di essere nominato medico statale per un periodo di sei mesi con lo stesso salario che aveva il chirurgo Gianbattista Vanucci. ${ }^{19}$

14 J. TADIČ, Jevreji u..., op. cit., p. 249; DAD, Lamenti politici, XI, vol. 5, fol. 164.

15 DAD, Acta Consili Rogatorum (Act. Cons. Rog.), III, vol. 54, fol. 121'.

16 DAD, Diversa di Cancelariae ( Div. Canc.), XXV, vol. 142, fol. 51.

17 DAD, Div. Canc., XXV, vol. 142, fol. 102'.

18 DAD, Cons. Min., V, vol. 45, fol. 48: "Captum est de precipiendo Domino Amatto Hebreo, medico, non posit in civitate nostra exercere officium medici si reverendiismum Dominus Archiepiscopus noster sibi non concesserit."

19 DAD, Acta. Cons. Rog., III, vol. 54, fol. 133'. 


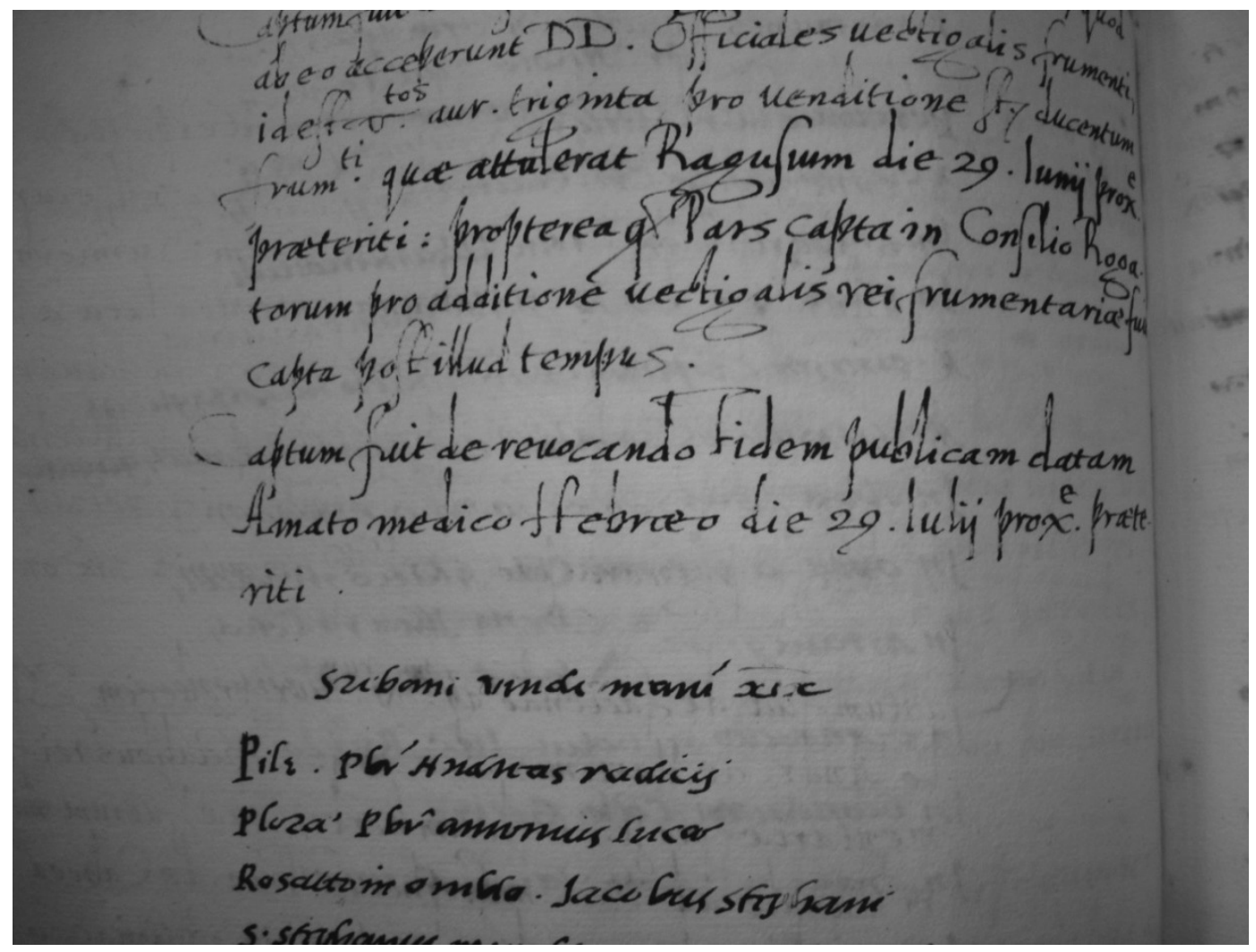

DAD, Acta. Cons. Rog. III., vol. 54, fol. 133', L'autorizzazione del medico Amatus Lusitanus

La mozione del governo ebbe 23 voti in favore e 12 contrari. Il detto risultato mostrava la popolarità di Amatus a Dubrovnik. Due giorni più tardi il Minor Consiglio deliberò favorevolmente in favore di Amato l'ebreo, accordandogli salario dei tre mesi pregressi, in conformità alla decisione presa in seno al Consiglio dei Pregati. ${ }^{20}$ Era evidente il tentativo di cooptare Amato nel servizio statale, e tuttavia questi non ottenne il necessario permesso dell'Arcivescovo. Fu così che il 2 maggio 1558 si avviò il dibattimento al fine di decidere subito ovvero rimandare la decisione, trattando nel frattempo con l'Arcivescovo. Con 20 voti in favore e 15 contrari fu accettata la proposta di dibattere l'accoglimento o meno del Lusitano presso il servizio statale. Fu deciso con 27 voti a favore e 8 contrari di revocargli il servizio e la paga, peraltro già approvata, visto che mancava il permesso dell'Arcivescovo quando fu accolto nel servizio. Cosi Amatus fu dimesso e alcuni cittadini si impegnarono per un intervento presso la Santa Sede

20 DAD, Cons. Min., V, vol. 44, fol. 58'. 
a suo favore, evidenza che fa dedurre la stima generalizzata di cui godeva per il suo mestiere. Un gruppo di cittadini fece appello al governo di adoperarsi presso il Papa, ma il Senato con 20 voti favorevoli e 19 contrari decise di non implicarsi direttamente nel caso. ${ }^{21}$ Questa decisione era un messaggio chiaro che vi erano due gruppi contrastanti e che una corrente politica si opponeva alla sua nomina. Va notato che a quell'epoca l'arcivescovo di Dubrovnik era Lodovico Beccadelli (1555-1560), umanista famoso di Bologna, amico di Michelangelo Buonarotti. La sua partenza da Dubrovnik diede occasione al poeta Savino Bobaljević, paziente di Amatus, di comporre un sonetto in onore dell'arcivescovo. ${ }^{22} \mathrm{E}$ singolare che Amatus Lusitanus non ottenesse il permesso dell'arcivescovo, doveva pur esserci una ragione importante. Dobbiamo tenere conto che il Senato di Dubrovnik, dietro pressione di Ivan Gundulić (Gondola), avesse sollecitato la nomina del Beccadelli prima al papa Giulio III e poi a Marcello II. Amatus era medico della figlia di Ivan Gundulić. ${ }^{23}$ Si tratta di Marija Gundulić, moglie del famoso filosofo Nikola Gučetić (Gozze), amica della potessa Flora-Cvijeta Zuzorić. Marija Gundulić in quell'epoca era una delle donne più erudite di Dubrovnik. Può darsi che una delle ragioni della nomina di Beccadelli fosse l'elezione di papa Paolo IV, grande amico della Repubblica di Dubrovnik e di Bonifacio Stjepović dell'isola di Lopud, custode della Terra Santa, il quale aveva introdotto alcune misure restrittive riguardanti la vita degli ebrei. Può darsi però che una delle ragioni dell'interesse di Amatus per la botanica lo spinse a criticare il rinomato umanista e medico Pietro Andrea Mattioli. Il secondo libro dei commenti al Dioscoride, pubblicado a Venezia, nel 1553, fu dedicato al Senato di Dubrovnik, scritto appunto quando Amatus voleva venire a Dubrovnik. ${ }^{24}$ Il noto medico raguseo Toma Budislavić (1545-1608) studiava a Bologna e Padova ed era amico del poeta Didaco Pirro del quale parleremo più estesamente nella seconda parte di questo lavoro. A Dubrovnik visse anche Giacomo di Lorenzo Sorkočević (1534-1604) collaboratore del naturalista Ulisse Aldrovandi e del segretario Antonio Gigante, segretario dell'arcivescovo Lodovico Beccadelli. Il patrizio raguseo Giacomo di Lovro Sorkočević senza dubbio conosceva Amato e curava i membri della sua stessa famiglia. Egli scambiava la conoscenza botanica, pesci e molluschi della regione di Dubrovnik, con il professore dell'Università di Bologna. A Dubrovnik era conosciuto il lavoro del medico e botanico Mattioli e il suo

21 DAD, Acta. Cons. Rog., III, vol. 54, fol. 150.

22 Daniele FArLatı, Illyricum sacrum, vol. VI. Venezia, 1800, pp. 230-242; Mijo BrLEk, "Lodovico Beccadelli", Hrvatski biografski leksikon, sv. I. Zagreb, 1983, pp. 562-565; Stjepan Krasıć e Serafino Razzı, Povijest Dubrovačke metropolije i dubrovačkih nadbiskupa (x. - xvı. stoljeća). Dubrovnik, Biskupski ordinarijat, 1999, pp. 141-144.

23 Lavoslav Glesinger,"Dvije studije o Amatusu Lusitanusu", Židov, vol. I, 20 (1937), p. 83.

24 In Dioscoridis Anazarbei de medica materia libros quinque enarrationes eruditissimae doctoris Amati Lusitani medici ac philosophi celeberrimi, quibus non solum officinarum Seplasiariis, sed bonarum etiam literarum studiosis utilitas adfertur, quum passim simplicia Graece, Latine, Italice, Hispanice, Germanice et Gallice proponantur. Venetiis, [apud Gualterum Scotum], 1553. 
commento all'opera del botanico greco Dioscoride. Pietro Andrea Mattioli nacque il 23 marzo 1501 a Siena e morì 1577 a Trento. Studiò a Padova dove compì gli studi nel 1523 ed esercitò la professione medica a Siena, Roma, Trento e Gorizia, diventando medico personale del Granduca Ferdinando II a Praga e poi di Massimiliano II a Vienna. Nella storia della scienza è noto piuttosto per il suo interesse per la botanica, dato che ha descritto 100 nuove specie nella botanica medica, oppure per l'applicazione medica. Ha esposto le sue conoscenze nel libro I Discorsi di M. Pietro Andrea Mattioli Senese, Medico Cesareo... Di Pedacio Dioscuride Anazarbeo Sulla materia medica (...). La prima edizione del libro di Mattioli fu pubblicata nel 1544 in italiano, dopo di che seguirono molte edizioni in latino, la prima, nel 1554 insieme a varie traduzioni. Amatus Lusitanus criticò fortemente Mattioli nelle sue Enarrationes che furono pubblicate per la prima volta nel 1553. A questa critica il Mattioli replicò con l'Apologia adversus Amathum Lusitanum cum Censura in eiusdem enarrationes, ${ }^{25}$ pubblicata per la prima volta nel 1558 a Venezia come un'opera a se stante, poi nello stesso anno e più volte insieme all'edizione latina dei commenti del Mattioli al Dioscoride. In questa opera Amatus fu accusato di non attenersi ai dogmi della fede cattolica, ma di essere rimasto fedele alla fede giudaica. Mattioli era una persona autorevole ed importante - medico personale del Granduca austriaco, sicchè Amatus procacciò, criticando la sua opera, senza dubbio un nemico pericoloso. La situazione creatasi fu certamente nota al ceto nobile raguseo. Riflettendo sulla prassi di Amatus a Dubrovnik, bisogna prendere in considerazione la situazione politica e la conoscenza medica dell'epoca. Tra i pazienti di Amatus c'erano dei rettori della Repubblica con le loro famiglie. Così nel novembre 1559 il rettore era Pavao di Junije Gradić (Gradi). ${ }^{26}$ I membri del Consiglio Minore per l'anno 1558 erano: Jeronim di Junije Gradić, Martolica di Ivan Kabužić (Caboga), Petar di Luka Lukarević (Luccari), Đuro di Ivan Gučetić (Gozze), Nikola di Leon Đorđić, Junije di Miho Bobaljević, Dragoje di Martolica Crijević ${ }^{27}$, Marin di Damjan Menčetić (Menze), Toma di Jeronim Sorkočević, Lucijan di Jeronim Bunić (Bona) e Stjepan di Marin Džamanjić (Zamanja, Giamagna). ${ }^{28}$ Tra i membri del Minor Consiglio c'erano pure i pazienti di Amatus. Tra i membri del Consiglio dei Pregati durante il mese di aprile c'erano Ivan di Marin Gučetić, Pavao di Marin Gučetić, Marin di Frano Gučetić e Frano di Ivan Kabužić. ${ }^{29}$

Amatus rimase a Dubrovnik ancora per un certo tempo e partì per i dintorni di Dubrovnik, da dove intendeva andare a Venezia. Per essere sicuro chiese al governo raguseo la "fidem publicam" per un periodo di quindici giorni dopo l'arrivo a Dubrovnik, per poter poi trasferirsi

25 J. TADIĆ, Jevreji u..., op. cit., p. 284.

26 DAD, Leges et instructiones, XXI.1., vol. 2, fol. 12.

27 DAD, Div. Canc., XXV, vol. 142, fol.12.

28 DAD, Leges et instructiones, XXI.1., vol. 2, fol. 27.

29 DAD, Leges et instructiones, XXI.1., vol. 2, fol. 70'. 
a Venezia. Il governo accettò la sua richiesta il 29 giugno $1558^{30}$, ma qualcuno si ribellò di nuovo contro Amato sicchè lo stesso governo il 12 agosto ritirò la "fidem publicam". ${ }^{11}$ Si trattava evidentemente di una corrente contraria esistente a Dubrovnik. Nel frattempo, l'8 luglio le autorità ragusee stavano rinnovando accordi con tre medici ed esprimevano l'interesse per un altro medico a due anni ulteriori. Per qualche motivo nascosto Amatus non era desiderabile. Si trattava evidentemente di un'atteggiamento volto a evitare disgrazie delle autorità spagnole, ma anche di dimostrazione della propria neutralità fra i Turchi e le potenze cristiane in quel tempo molto delicato. ${ }^{32}$ Dubrovnik aveva una relazione diplomatica molto amichevole con l'imperatore Carlo V (1519-1558), ma di nascosto dava comandi ai suoi capitani nei porti spagnoli per non prendere parte nella flotta spagnola nelle loro lotte contro gli Ottomani, essendo continuamente accusata da parte dei diplomatici inglesi e francesi alla corte ottomana di essere alleati spagnoli. ${ }^{33}$ Il Lusitano abitava a Dubrovnik in un momento in cui la città era un centro di spie, quando a causa di violenti incursioni turche di anno in anno cadevano, uno dopo l'altro, pezzi del regno di Croazia. Era questa una situazione molto grave nella quale Dubrovnik, isolata ed esposta a permanenti pericoli, cercava di difendersi. Tra le spie spagnole vi era il più noto Marino Giamagna, patrizio raguseo e titolare dell'ordine equestre di S. Giacomo; le lettere di questo delatore sono conservate nell'Archivio General de Simancas riferite a tre periodi, dei quali il medio copriva gli anni 15541562.

I membri della comunità sefardita a Dubrovnik prestavano servizi informativi a diversi Stati. Uno di questi, David Paz (Passi) offriva informazioni agli Ottomani. ${ }^{34}$ La partenza di Amato da Dubrovnik coincise in ogni modo con i preparativi del pascià Piala per occupare la fortezza a Minorca nell'anno 1559. L'atteggiamento di sfiducia nei confronti dei "marrani" e "moriscos" era una conseguenza della sfiducia della Corte spagnola. ${ }^{35}$ Amatus partì verso la fine del 1558

30 DAD, Cons. Min., V, vol. 44, fol. 84: "Fides publica Captum fuit de dando Fidem publicam Amato medico per dies xv; postquam ingressus fuerit Ragusiam; ut hinc possuit traduci Venetias."

31 DAD, Cons. Min., V, vol. 44, fol. 88': "Captum fuit de revocando Fidem publicam datam Amato medico Hebreo die 29. lulij prox.e prateriti."

32 Robin Harris, Povijest Dubrovnika. Zagreb, Golden marketing-Tehnička knjiga, 2006, p. 103.

33 R. HARRIS, Povijest..., op. cit., pp. 100-102.

34 Ari BüLENT, "1. Appendix to the text "An Influental Jew on the Ottoman Court: David Pass": in Mirjana Polıć Bobıć (ed.), Tajna diplomacija u Dubrovniku u xvı. Stoljeću. Zagreb, Sveučilište u Zagrebu, 2011, pp. 194-198.

35 Mirjana Polıć Bobıć, Među križem i polumjesecom, dubrovačke dojave španjolskom dvoru o Turcima u xvı. Stoljeću. Zagreb, Naklada Ljevak, 2000, pp. 1-96; idem, "Activities of the Spanish Secret Service in the Republic of Dubrovnik in the $16^{\text {th }}$ Century and the Role of Spanish Confidant Marin Zamanja", in Mirjana Polıć Bobıć (ed.), Tajna diplomacija u Dubrovniku u xvı. Stoljeću. Zagreb, Sveučilište u Zagrebu, 2011, pp. 181-190; idem, "Španjolska tajna služba u Dubrovačkoj Republici u xvı. stoljeću i uloga španjolskog povjerenika Marina Zamanje u njoj", in Mirjana Polıć Bobıć (ed.), Tajna diplomacija u Dubrovniku u xvı. Stoljeću. Zagreb, Sveučilište u Zagrebu, 2011, pp. 86-93. 
verso Salonicco e lì morì dieci anni più tardi come vittima della peste. Secondo la tradizione sulla sua tomba campeggiava l'epitaffio di quattro distici composti da Didaco Pirro. ${ }^{36}$

\section{L'ATTIVITÀ MEDICA DI AMATUS A DUBROVNIK}

L'opera più importante di Amatus sono le sette centurie, vale a dire le 700 più interessanti storie della sua attività professionale, accompagnate da spiegazioni supplementari — scholia. Per quanto ci riguarda, la più interessante è la sesta centuria (detta Ragusina), pubblicata per la prima volta a Venezia nel 1560, contenente cento casi pratici capitatigli a Ragusa, che ben illustravano le condizioni culturali e sanitarie a Dubrovnik in epoca rinascimentale. A Dubrovnik Amatus fu il primo a introdurre erbe medicinali di origine orientale (radix sinarum), e lottava anche contro la superstizione. ${ }^{37}$ I casi più interessanti e importanti della sua prassi medica Amatus li studiava facendone appunti e protocolli, ottenendo così un grande numero di dati scientifici relativi alle malattie e ai pazienti della sua decennale attività. In tutto ve n'erano sette centurie, alcune delle quali pubblicate più volte. Prima del suo arrivo a Dubrovnik aveva pubblicato a Venezia nel 1557 solo quattro centurie, altre tre le pubblicò più tardi avendo messo in ordine gli appunti corrispondenti. La Quinta Centuria fu scritta ad Ancona e poi rivista e completata durante il soggiorno a Dubrovnik, mentre la Sesta Centuria contiene appunto i cento casi della sua prassi a Dubrovnik. Ambedue furono poi stampate a Lione nel 1564. A Salonicco ha scritto l'epilogo nel 1559 e il proemio nel 1560. Dedicò questa edizione a Joseph Naci. L'opera inizia con un incontro fra tre patrizi ragusei: Giovanni Gradi, Simone Benessa e Pasquale Gozze, i quali guidano Amato presso un patrizio malato, Medo (Orsatto) Gundulić. Segue una breve descrizione di Dubrovnik. Si constata che la città fosse esposta ai forti venti del sud, per quanto durante l'inverno la città fosse colpita dal passaggio di molte malattie; inoltre mancava il frumento, data l'assenza di pianure, mentre il vino è aspro e insalubre. La città è governata da nobili, abili politici che si occupano del commercio, ma anche da semplici cittadini. I popolani sono poveri. Amatus fa una descrizione a parte per ogni caso: in primo luogo narra di chi si tratta e in che modo si è ammalato. Poi descrive la degenza, e la ricetta specifica prescritta, aggiungendovi spesso anche annotazioni (scholia) riferiti a medicine antiche, greche e arabe. Singoli casi sono descritti piuttosto brevemente, a differenza dell'ultimo caso nel quale presenta la cura di un capitano marittimo ferito alla testa da un marinaio. Il caso

36 J. TADIČ, Jevreji u..., op. cit., 1937, p. 297; Diogo PIREs, Cato Minor siue Disticha Moralia ad Ludimagistros Olysipponenses (...). Venetiis, apud Felicem Valgrisium, 1596, p. 163.

37 Vladimir DuGaČKI, "Amatus Lusitanus", Hrvatski biografski leksikon, vol. I. Zagreb, 1983, p. 237; Risto Jeremić e Jorjo TAdić, Prilozi za istoriju zdrastvene kulture starog Dubrovnika, vol. 2. Beograd, 1939, pp. 89-124; L. Glesinger, "Amatus...", op. cit., pp. 61-62; idem, "Dubrovački...", op. cit., pp. 106-107. 
è descritto in maniera drammatica alla stessa stregua di quello introduttivo: un certo patrizio della famiglia Gradi prega il Lusitano di ricevere il suo aiuto. Accanto a lui stanno due farmacisti ragusei (Celentano e Vanucci) con i quali Amatus discute e prescrive che cosa bisogna fare. Il libro finisce con Amatus (Amati iusiurandum), che giura su Dio e Mosè ecc. per aver sempre cercato di giovare agli infermi e non a se stesso, di non aver mai guardato chi è ebreo, cristiano o musulmano, di non aver aiutato i farmacisti più di altri, di non aver mai scoperto un segreto affidatogli, né dato veleno a chiunque, né assistito a qualche aborto. Il giuramento è scritto con parole molto serie ed emozionanti. Secondo l'opinione di Amato molte malattie sono causate dal vento del sud, mentre il vino cattivo causava molti casi di calcoli vescicali. D'altra parte sosteneva che a Dubrovnik le ferite alla testa cicatrizzano facilmente. Oltre a Dubrovnik Amatus descrive anche la situazione sanitaria della città di Ston (Stagno), dove andava per curare i casi di grave febbre paludare. Amatus curava pure i patrizi che soffrivano dalle malattie veneree. Le centurie ci presentano Amato come un ottimo medico clinico, molto dotto, teorico e pratico della sua epoca. Conosceva bene la letteratura specifica e si occcupava di anatomia. Era pure un esperto di chirurgia, un buon diagnostico e terapista esperto. ${ }^{38}$ Nella sesta Centuria ${ }^{39}$ Amatus presenta la lode a Dubrovnik (p. 264): "Ragusii civitatis brevis descriptio, per Amatum Lusitanum. Ragusium, urbs parua, sed antiqua, et Venetiarum simia, ad mare Illyricum inter rupes sedet, $\&$ meridiem spectat (...)”. L'opera è molto importante per la conoscenza della storia culturale di Dubrovnik. Oltre ad aspetti geografici vi si trova anche una succinta valutazione del governo: "Il governo è repubblicano, ma vi si ammettono solo i nobili, i quali sono abili politici, uomini abbastanza ricchi e sensati. Oltre ai nobili vi è una moltitudine di cittadini, una parte dei quali si occupano di commercio. Questi sono abbastanza colti e le loro navi sono bellissime. L'altra parte dei cittadini vive in miseria e povertà.” Da queste parole emerge un'immagine meno nota della Dubrovnik rinascimentale. ${ }^{40}$

Tra i pazienti di Amatus vi erano numerose persone di Dubrovnik: Jeronim Binčulić (Hieronimus Bucignolius, p. 265), figlio di Benedikt Gundulić (p. 270), Marko di Jero Basiljević (Marcus filius Hieronimi Basilii, p. 272), Nikola di Vlaho Držić (Nicolaus, filius Blasij Darsae, p. 272), Marin Gradić (Marinus Gradius, patritius raguseus, p. 272 e 285), filius Sacrintoris Ragusini (p. 288), aurifex Racusinus (p. 298), figlio di Nikola Bunić (Filius Nicolai Bonij, patritij ragusaei, p. 289), Dominik Kladorubović, mercante raguseo (p. 291), figlio di Nikola Palmotić (filius Nicolai Palmotae Patritii Ragusaei), figlio di Pietro Katarini (filius

38 J. TADIČ, Jevreji u..., op. cit., pp. 291-295; Milan ReŠETAR, "Amat Luzitanac, dubrovački ljekar xvı. Vijeka", Brankovo kolo 6 (1900), pp. 39-41; Lavoslav Šik, Die jüdische Ärtze in Jugoslavien. Osijek, E. Sekler, 1931, pp. 9-20; B. KREKIČ, "The role...", op. cit., p. 269.

39 Amatı Lusitani, Curationum medicinalium, Centuriae duae, Quinta et Sexta (...). Lugduni, apud Gulielmum Rouillium, 1564, pp. 262-647.

40 L. Glesinger, "Amatus...", op. cit., pp. 61-62. 
Petri Catarini, p. 293), Jakov di Nikola Bunić (Jacobus, filius alterius Nicolai Bonii Patritii Ragusei, p. 297), moglie di Mudai (vxor Mudai, p. 298), Frano coniatore raguseo (Franciscus nummularius Racusinus, p. 300), la donna turca Imin da Durazzo (Turca Imin, ex Duratio, p. 301), Pavao Gradić (Paulus Gradius, patritius Ragusaeus, p. 303), figlia di Dominik Kladorubović, mercante raguseo (filia Dominici Cladorouichi, mercatoris Racusini, p. 306), Marin Gundulić (Marinus Gondullanus, nobilis iuuenis Ragusanus, p. 308), Stjepan Menčetić (Stephanus Maenssius, patritius Racusinus, p. 314), Sebastijan Bobaljević (Sebastianus Bubalius ex Racusaeis patritiis, p. 320), figlio di Manuele Fragosi (Filius Manuelis Fragosii, p. 325), Sjepan Ranjina (Stephanus Aranius ex patritio Ragusaeorum, p. 331), Didaco Pirro (p. 334$)^{41}$, Pavao Gradić il giovane (Paulus Gradius iuuenis, Patritius Ragusaeus, p. 338), suo nipote Junije Gradić (Junius Gradius, Patritii praedicti nepos, p. 345), moglie del mercante raguseo Đuro Martinić (vxor Georgii a Martinis, ciuis Ragusini (p. 350), moglie di Miho di Scipione Bunić (vxor Michaelis Scipionis Bonii, ex patritia Ragusinorum familia, p. 351), mercante Salonicense Krispin (p. 360), moglie di Božo Prokulić (Vxor Natalis Proculei, patritii Ragusini, p. 368), Giacomo francese ed Enrico francese (Jacobus homo Gallus \& Henrici Francorum Regis, p. 373), Thamir, mercante turco da Pergamon (p. 378), Nikola Gučetić (Nicolaus Gozius, ex nobili Ragusaeorum familia, p. 381), figlio di Zmaj (Draco) Crijević (Filius Draconis Ceruini, patritii Racusini, p. 386), Šimun Beneša (Simeon Benesius, vir doctus, ex nobilibus Ragusaeis, quem e Byzantio, ubi Legatum ad Solimanum regem pro patria egerat, p. 386), Ivan Lukarević (Ioanni Luce, viro probo \& patritio raguseo, p. 396), figlia di Nikola Sorkočević (ex patritia Ragusaeorum familia puella filia Nicolai Sorgij, p. 400), Aloisia, moglie del capitano Dalmata (Aloisia, Dalmatiae Nauarchi uxor, p. 410), Luka Buća (Luca Bucchius, nobilis Ragusaeus, p. 414), Marin Menčetić (Marino Machullio, iuuenis nobilis, p. 416), madre di Frano Sorkočević (mater Francisci Sorgii, p. 418), Andreas Myroparonis Praefectus (p. 429), moglie di Vlaho di Valentin Sorkočević (uxor Blasii Valentini Sorgii, p. 434), Pavao Gradić il maggiore (Paulus Gradius Senior, p. 438), Bernard Bunić (Bernardus Bonius, p. 442), moglie di Abner Alfarin (uxor Abner Alpharini Haebraeorum, p. 443), Bernard, abbate dell'abbazia benedettina di Lacroma (Bernardus Abbas ex Croma insula, p. 444), Abner Alpharinus Haebraeus console (Abner Alpharinus Haebraeus, p. 447, apud Ragusaeos consul), Marin Getaldić (Marinus Guetaldus, ex Ragusaeorum patritia gente, p. 449), Matija Paskalić (Mattheo Paschali nobilis Catharensis, p. 457), figlio di Petar Lukarević (filius nobilis Petri Luchari, p. 461), Marin Gundulić (Marinus Gondulanus, p. 463), Jakov Basiljević (Jacobus Basilius, nobilis Ragusaeus (p. 466), Alojzije (Luigi), mercante raguseo (Aloisius Ragusaeus mercator, p. 470), figlia di Petar Lukarević (filia Petri Luchari, nobilis Ragusaei, p. 475), Stjepan Gučetić (Stephanus

41 "Curatio trigesima, in qua agitur de dolore iocinore. Didacus Pyrrhus, vir Graece et Latine peritissimus, et magni nominis poeta. Cum in suburbio Ragusii ageret". 
Gozius, nobilis \& magnificus Ragusaeus, p. 481), Josip Gučetić (Josephus Gozius, nobilis Ragusaeus, p. 485), madre di Junije Đorđić (p. 487), Marin isolano (Marinus vir insularis, p. 496), Lodovico Spadaio (Ludouicus Spatarius, p. 499), Enea Napolitano musicista (Aeneas Neapolitanus musicus, p. 508) e Marin Sorkočević (Marinus Sorgius, nobilis Ragusaeus, p. 531) e numerosi altri.

Leggendo questi nomi con l'indicazione delle rispettive malattie, Amatus Lusitanus si pone una domanda di tipo etico. Ciononostante, il Lusitano ha fornito un'idea interessante sulla situazione a Dubrovnik del Cinquecento, conosciuta come "epoca aurea di Dubrovnik". Conoscendo i nomi dei pazienti di Lusitano, presso l'Archivio di Stato di Dubrovnik, possiamo trarre numerose conclusioni sull'esito delle cure e sulle sorti dei patrizi e cittadini di Dubrovnik di quell'epoca. Per esempio dal caso di Nikola, figlio di Vlaho Držić, mercante e pittore raguseo (Dubrovnik, 20 luglio 1503 - Dubrovnik 1569), o da quello della famiglia Držić (Darsa) dalla quale provengono parecchi personaggi illustri, artisti ed architetti militari. Vlaho di Marino Držić, mercante e pittore, era fratello maggiore del commediografo Marino, che sin dalla gioventù visse a Venezia, occupandosi di commercio e arte, sposato con una veneziana. Dopo il fallimento dei suoi affari nel 1541 decise di tornare a casa dove tre anni dopo sarebbe entrato al servizio statale quale scrivano per l'arte laniera. Fungeva pure da esperto delle opere d'arte. Come tale fece valutare la qualità artistica di un altare della chiesa di S. Andrea. Nel 1548 firmò un contratto per la realizzazione di una pala d'altare per il nobile Ilija Bunić. Ad ogni modo, Vlaho Držić era un personaggio particolare: teneva rapporti di amicizia con diversi umanisti italiani, fra i quali Antonio Brucinolo e Pietro Aretino. Apparteneva alla cerchia artistica intorno al famoso pittore Tiziano Vecellio coltivando pure rapporti di amicizia con il pittore Giovanni de Ponte, figlio del conosciuto pittore veneziano Giacomo de Ponte. Il famoso matematico, astronomo, commediografo e poeta Nikola Nale-Nalješković (+1587) stenta a trovare le parole adeguate per elogiare il globo adornato da Držić per il cardinale Carapi, e ne risaltava tuttavia la sua spiccata capacità nel ritrattiare i soggetti. Il poeta Michele Monaldi, suo compagno di gioventù a Firenze, compose una poesia in cui loda Držić che con i suoi colori dava vita alla tela, dimostrando di che cosa fosse capace l'arte. Gli si attribuiscono, senza ragione, due pale di altare nella chiesa di s. Domenico a Dubrovnik: la Madonna con i santi Biagio e Francesco e la pala di Sacra Famiglia nella chiesa della Madonna di Šunj sull'isola di Lopud. Alcuni suoi biografi ritengono che era solo un autodidatta di talento, altri invece negarono le qualità artistiche lodate dai suoi contemporanei ineguagliate da nessun'altro pittore di Dubrovnik. Altri sono del parere che egli avrebbe potuto imparare l'arte pittorica durante il suo soggiorno in Italia lavorando presso qualche pittore rinomato. Ad ogni modo, nel 1603 Držić viene menzionato nella Genealogia della fraternità degli Antonini dove esercitava l'arte pittorica e scultorea e realizzava opere lodevoli. Lo storico Serafino Crijević (Cerva) scrive che era un uomo di mano molto abile, un artista universale e collezionista di opere d'arte antiche. 
Si tratta senza dubbio di un personaggio di talento, tipicamente rinascimentale. Egli è spesso menzionato dagli scrittori coevi: Antonio Brucioli fiorentino vissuto a Venezia quale esule politico lo ricordava nei suoi dialoghi platonici; Pietro Aretino invece, in una lettera del 1545, lo ringraziava per avergli regalato una scatola utile a conservare pettini, elaborata artisticamente, ne lodava le opere ("meravigliose opere ch'escono da voi”, "mirabili lavori”); il poeta Nicola Nale-Nalješković, oltre ad elogiare il globo dorato realizzato per il cardinale Carpi, menzionava anche le doti prospettiche e l'arte di damascare. Il poeta Michele Monaldi, compagno di gioventù a Firenze, in un'epistola a Savino Bobaljević (Bobali) - Mišetić, in una poesia intitolata "In morte del Drusiano" faceva riferimento alla sua morte. Padre di Nicola, paziente di Amato, era Biagio, fratello di Marino Držić (Dubrovnik 1508-Venezia 1567). Durante il soggiorno di Amato a Dubrovnik Marino scrisse diversi drammi e fece una traduzione di Ecuba di Euripide. ${ }^{42}$ La causa della morte prematura di Nicola (1530-1567), che era sordomuto, fu probabilmente la malattia. ${ }^{43}$ Amatus scrive di aver curato anche la moglie di Abner Alfarino, mercante, armatore e console a Dubrovnik. Nel 1559 il console Abner vendette una nave al patrizio raguseo Scipione di Frano Kabužić (Caboga). ${ }^{44}$

42 Franjo Švelec, "Marin Držić", Hrvatski biografski leksikon, vol. III. Zagreb, 1993, pp. 631-636; idem, "Vlaho Držić", Hrvatski biografski leksikon, vol. III. Zagreb, 1993, pp. 636-637.

43 Nenad VekARIČ, Vlastela grada Dubrovnika, 4. Odabrane biografije (A-D). Zagreb-Dubrovnik, Zavod za povijesne znanosti HAZU u Dubrovniku, 2013, p. 347.

44 J. TADIČ, Jevreji u..., op. cit., p. 226. 


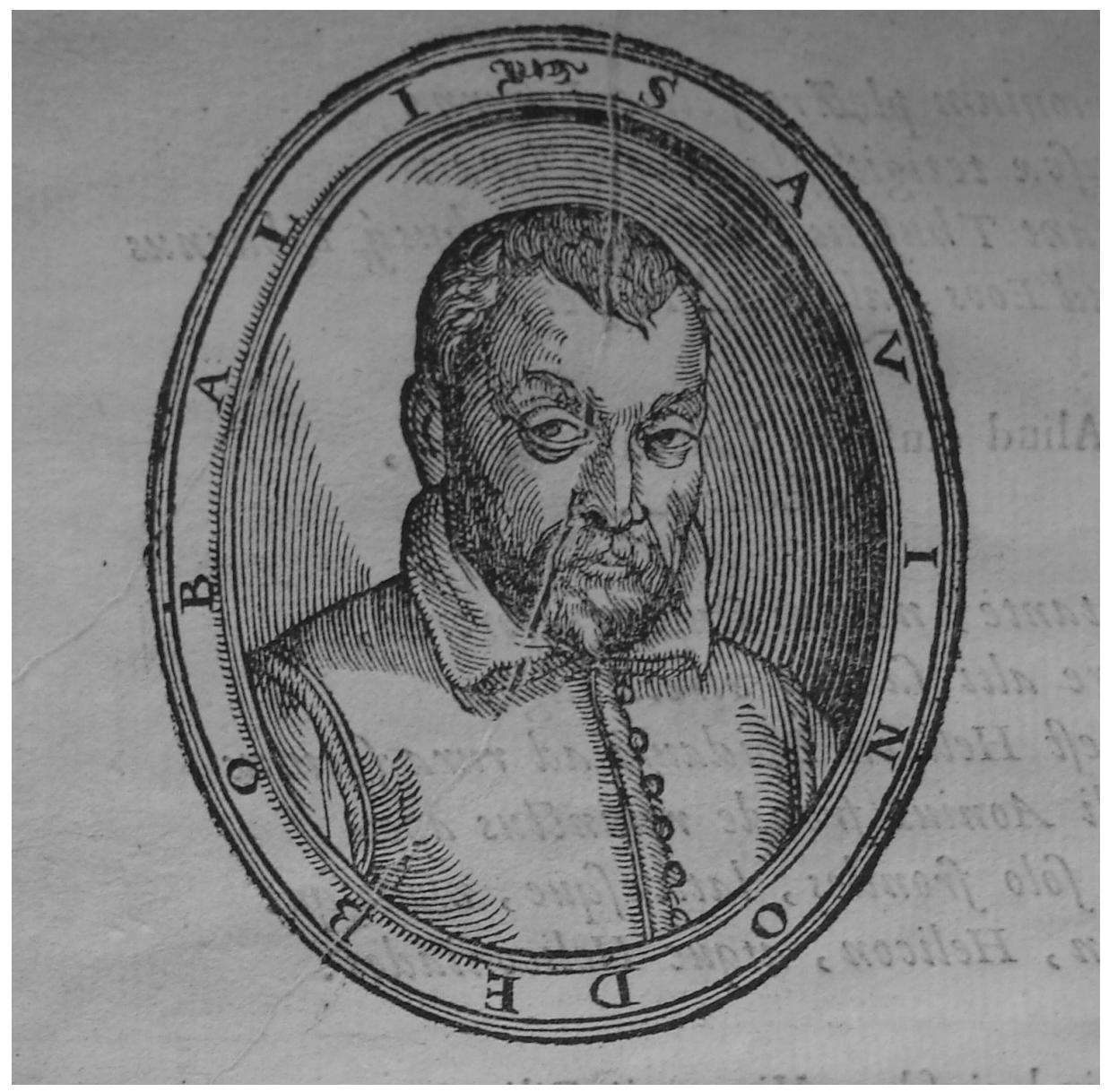

Il ritratto di Savino (Sabo) di Miho Bobaljević (1530-1585) da suo canzoniere Rime amorose, e pastorali, et satire, Venezia, 1629.

Savino (Sabo) di Miho Bobaljević (1530-1585) soprannominato il Sordo, scriveva in italiano e in croato. Il suo canzoniere "Rime amorose, e pastorali, et satire" fu stampato a Venezia (quattro anni dopo la sua morte) dai suoi fratelli Sigismondo e Marino. Nel canzoniere ci sono sonetti dedicati a diversi notabili italiani: Anibale Caro, Laura Battiferri, Domenico Venier, Benedetto Varchi, Lodovico Beccadelli e altri. Altri sonetti sono dedicati ai suoi contemporanei, suoi modelli e amici, poeti: Mavro di Dominik Vetranović (1482-1576), poliglotta Miho di Marin Menčetić - Matufić (ca 1523-1590), filosofo e poeta Matteo di Damiano Benessa (ca 1530-1599), scrittore giuridico Simone di Mateo Beneša (cca 1525-1596), a Marino di Marino Držić (1508-1567) e molti altri. Le sue poesie scritte in croato sono quasi tutte andate perdute. 
È conservato, invece, il "Lamento di Arianna” scritto in stile di Ovidio, versione libera dell'Amor fuggitivo di Tasso, la zingaresca Jeđupka e alcune poesie amorose. Sono pure conservate le sue epistole, le più importanti sono quelle dirette ai poeti Maroje Mažibradić e Mikša Pelegrinović. Egli era probabilmente il fondatore dell'Accademia dei concordi. Il poeta Antonio Sassi-Sassin lo elogia nelle poesie "Drugi san" (Altro sonno) e "U pohvalu pjesnika dubrovačkijeh" (In lode dei poeti ragusei). Un'epitafio in suo onore fu scritto da Dominik di Šimun Zlatarić (ca 1557-1613). Un altro epitafio gli fu dedicato da Miho di Marino Bunić/Bona - Babulinović (ca 1551-1617) e Michele Monaldi (“In morte del Sig. Savino Bobalio il Sordo").45

Era paziente di Amatus anche un figlio di Dragoje Crijević/Cerva, molto probabilmente Martolizza (ca 1537-1608), durante la cura aveva circa venti anni. Suo fratello Orsatto era un notabile diplomatico che difendeva Dubrovnik dalle accuse degli ambasciatori francesi e inglesi di aiutare gli spagnoli in funzione anti turca. Da 1599 fino a 1601 Orsatto fu alla corte del re di Spagna Filippo III la cui interposizione confermava i privilegi commerciali ragusei. ${ }^{46}$

Tra i pazienti di Amato c'era pure una figlia del mercante Dominik Kladorubović, discendente di un nobile bosniaco di Srebrenica. Domenico e sua moglie Deša, della famiglia di mercanti Radalj, ebbero parecchie figlie: Anica, Katarina, Frana (moglie di Šimun di Miho Zlatarić) e figli illegittimi: Jeronim, Secondo, Prudenzia e Nikola — benedettino vissuto sulle isole Tremiti. ${ }^{47}$ La paziente di Amato era probabilmente Anica ovvero Katarina, perchè Frana si era sposata nel $1555 .{ }^{48}$ Questa signora era madre di Dominik Zlatarić, giovane amico di Didaco Pirro il quale nel 1587 sposò Mara, quest'ultima plausibilmente figlia del pittore Pietro di Giovanni: la paziente di Amato, la cui cura è stata descritta, era la zia del pittore. ${ }^{49}$

Della casata Gundulić erano due pazienti di Amato: Marin Gundulić e figlio Benedikt Gundulić. Marino era probabilmente figlio di Ivan, nipote di Lujo (Luigi) di Dragoje Gučetić (Gozze), un ricco mercante di Ancona, noto per la commissione di una pala d'altare di Tiziano Vecellio per i francescani di Ancona. L’anonimo figlio di Benedik Gundulić potrebbe essere il figlio del ricco mercante di Ancona, in precedenza mercante raguseo a Londra. ${ }^{50}$

45 Nenad VeKARIČ, Vlastela grada Dubrovnika, 4..., op. cit., pp. 32-38.

46 Nenad VeKARIČ, Vlastela grada Dubrovnika, 4..., op. cit., p. 298.

47 DAD, Testamenta de Notaria, X.1., vol. per l'anno 1568, fol. 7; DAD, Descrizione delle origini e Genealogie dei Cittadini Ragusei che furono in affito della Confraternita di San Antonio nei tre Secoli e più da che comincia la medesima che fù l'anno 1343 e seguita poscia la sua real fondazione come per consentimento del E.mo Maggior Consiglio della Republica di Ragusa che fù l'anno 1348 visino a tutto (...), fol. 277-280'.

48 DAD, Pacta matrimonialia, per la data 26. IV. 1555.

49 DAD, Famiglie Cittadine riconosciute dall'Ex Repubblica di Ragusa e loro descrizione, e parentele, pp. 189-191.

50 Nenad VeKARIČ, Vlastela grada Dubrovnika, 2. Vlasteoski rodovi (A - L). Zagreb-Dubrovnik, Zavod za povijesne znanosti HAZU u Dubrovniku, 2012, pp. 312-313. 
Dalla famiglia patrizia Gradić c'erano parecchi pazienti del nostro. Tra questi Junije di otto anni. Si tratta di Junije di Mato Gradić (1549-1594), donatore del dipinto del maestro di Anversa. Marten de Vos alla chiesa di S. Rocco a Grgurići nel 1586, era un voto contro la peste. Il nome di Giounio si trova scolpito nel giardino del palazzo dei Gradi a Dubrovnik, e il poeta Frano Lukarević (Luccari) Burina (ca 1541-1590), gli dedicò la traduzione della commedia pastorale "Pastor Fido" di Giovanni Battista Guarini (1592). Pavao Gradić è probabilmente figlio di Marin di Junije, curato da Amatus come anziano (ca 1478-1558), dieci volte Rettore della Repubblica di Dubrovnik. Intraprese una corrispondenza letteraria con Marino Santo di Barletta sul significato delle comete. ${ }^{51}$

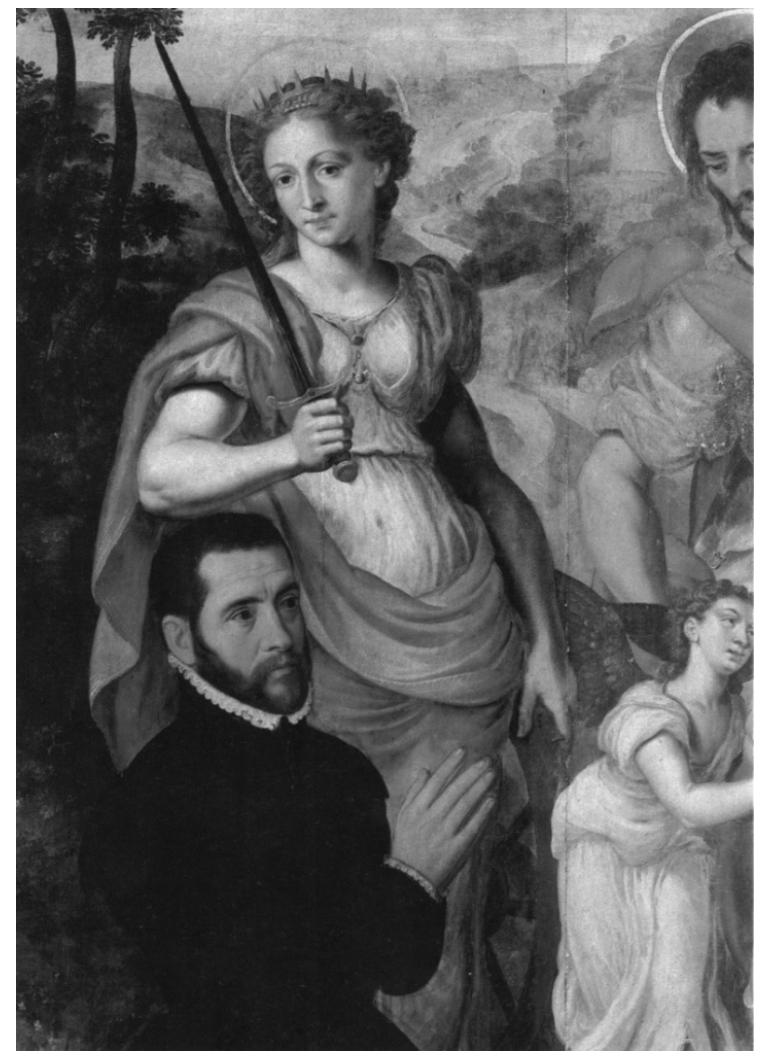

Marten de Vos, fiammingo pittore manirista (c. 1532-1603) dipinse la pala d'altare per la chiesa San Rocco in Grgurići vicino a Dubrovnik e sul quadro è il ritratto di Junije Matov di Gradić

51 Nenad Vekarıč, Vlastela grada Dubrovnika, 2..., op. cit., p. 281. 
Il paziente Marin Getaldić (Ghetaldi) era probabilmente Marin di Mato (ca 1499-1573) al tempo cinquantaquattrenne: da giovane nel 1595, quando fu conte a Trstenica, aveva scoperto e impedito una congiura dei Turchi per occupare la penisola di Pelješac (Sabioncello). ${ }^{52}$

Della famiglia patrizia Sorkočević (Sorgo) aveva come paziente moglie di Vlaho di Valentin (ca 1482-1591), mercante a Londra e quattro volte Rettore della Repubblica. Alle nozze di suo nipote Vlaho fu eseguita Grižula, la commedia del poeta Marino Držić, probabilmente nel $1556 .{ }^{53}$

Tra i più conosciuti pazienti di Amato a Dubrovnik era certamentte Šimun di Mato Beneša (ca 1525-1596) che nel 1554/5 era ambasciatore della Repubblica presso il re di Francia Enrico II e alla corte del Gran Turco a Costantinopoli. Durante quest'ultima missione nel 1563 fu accusato di essersi esibito con due cavalli e tre servitori e perfino di essere ceduto agli incantesimi di una donna turca. Nel 1591 egli scrisse un codice legale: "Praxis curiae ad firmam legum et consuetudinum Reipublicae Ragusinae". Savino di Miho Bobaljević gli dedicò due poesie e Didaco Pirro un poemetto. ${ }^{54}$

Il paziente di Amato era forse anche Nikola Gučetić (Gozze, 1549-1610), patrizio raguseo, membro delle Accademie perugine (degli Insensati e Occulti), autore di diverse opere filosofiche e letterarie: dialoghi platonici Della bellezza e Dell'amore, Discorsi su Meteori di Aristotele, Discorsi su sette Salmi penitenziali di Davide, Governo della famiglia, Dello stato delle repubbliche e Dialogo su immortalità e fortuna umana. L'intera opera di Nikola ebbe successo in diversi campi con le sue problematiche e rappresenta un contributo alla filosofia croata, in particolare alla riflessione estetica. È indubbio il suo contributo nel campo del neoplatonismo e neoaristotelismo rinascimentale. Il suo pensiero originale lo qualifica come precursore di nuove concezioni filosofiche. Anche sua moglie Maria si serviva dell'aiuto di Amato. ${ }^{55}$

Tra i cittadini delle regioni limitrofe curati da Amatus è menzionato il nobile di Cattaro Mato Paskalić (Pasquali), discendente di una nobile famiglia dalla quale proveniva anche il poeta croato Lodovico Pasquale (ca 1500-1551), già studente studiò a Padova, pubblicò in italiano una raccolta di testi intitolata Rime volgari di M. Ludovico Pascale da Cattaro Dalmatino, 1549, contenente 178 sonetti, 13 madrigali, 15 canzoni e numerose lettere. Una breve raccolta di rime in stile petrarchista con ventisei elegie fu pubblicata postuma dal suo amico e poeta italiano Lodovico Dolce (Ludovici Pascalis carmina ad Illustrissimum et Doctissimum

52 N. VEKARIČ, Vlastela grada Dubrovnika, 2..., op. cit., p. 154.

53 N. VeKARIČ, Vlastela grada Dubrovnika, 3. Vlasteoski rodovi (M-Z). Zagreb-Dubrovnik, Zavod za povijesne znanosti HAZU u Dubrovniku, 2012, p. 278.

54 N. VeKARIČ, Vlastela grada Dubrovnika, 4..., op. cit., pp. 96-97.

55 Nikola Vitov GuČetić, Dialogo della Bellezza/Dijalog o ljepoti i Dialogo d'amore/Dijalog o ljubavi. Zagreb, Matica hrvatska, 2008, pp. 1-321. 
Marchionem Austriae Bernardinum Bonifacium per Ludovicum Dulcium nunc primum in lucem edita. Venetiis apud Gabrielem Volitum et fratres de Ferrariis 1551). ${ }^{56}$

Da quanto detto, risulta che i pazienti identificati da Amato erano numerosi, soprattutto appartenenti all'alta borghesia e al patriziato di Dubrovnik e offrono molte informazioni sulle condizioni sanitarie e sociali dell'epoca a Dubrovnik.

\section{DIDACO PIRRO - DIOGO PIRES}

Didaco Pirro (Didacus Pyrrhus Lusitanus, ovvero Iacobus Flavius Eborensis), di nome vero Isaia Koen è un umanista portoghese e poeta scrittore latino. Nato a Evora il 5 aprile 1517 e morto a Dubrovnik il 16 maggio $1599 .{ }^{57}$ Si suppone che insegnasse in maniera privata alla gioventù ragusea, in particolare a coloro che frequentavano il ginnasio. ${ }^{58}$ Tuttavia tale ipotesi non trovano conferma dalle fonti archivistiche. È sicuro invece che le autorità ragusee gli commissionassero le traduzioni dei contratti commerciali dal portoghese ed ebraico in italiano, poi registrati presso l'ufficio notarile della Repubblica. ${ }^{59}$ Spesso era presente in occasioni di stipulazioni dei contratti commerciali. ${ }^{60}$ Visse in una casa privata presa in affitto sul litorale adriatico, poco prima di finire i suoi giorni nel ghetto. ${ }^{61}$ Le autorità ragusee ebbero una grande stima di lui, tanto da dispensarlo dall'obbligo di indossare il capello giallo o rosso. ${ }^{62} \mathrm{Nel}$ suo testamento Didaco Pirro lasciò due

56 Š. LJUBIĆ, Dizionario..., op. cit., pp. 239-240.

57 Bariša KREKIĆ, "The Role of the Jews in Dubrovnik (Thirtheen - Sixteenth Centuries)", in Bariša KREKIĆ, Dubrovnik, Italy and the Balkans in the Late Middle Ages. London, Variorum Reprints, 1980, pp. 257-271; idem, in Bariša KREKIĆ, Dubrovnik: a Mediterranean Urban Society, 1300 - 1600. Aldershot, Variorum, 1997, pp. 840, 842.

58 Toma KERŠA, Della vita e degli scritti di Didaco Pirro altrimenti detto lacopo Flavio Eborense. Commentario di Tommmaso Chersa. Firenze, nella stamperia Magheri, 1826; Antun PASARIć, "Pjesme Didaka Pira", Srđ, Dubrovnik, 1903, pp. 91-92, p. 141, pp. 184-186, pp. 215-218; Đuro KÖRBLER, "Život i rad humanista Didaka Pira Portugalca, napose u Dubrovniku", RAD JAZU, lib. 216 (1917), pp. 82-83; J. TADIć, Jevreji u..., op. cit., pp. 300-301.

59 DAD, Diversa de foris, vol. 3, fol. 131-133; Petar Kolendić, "Nekoliko pesama humaniste Didaka Pira", Zbornik istorije književnosti Odeljenja literature i jezika Srpske akademije nauka i umetnosti, lib. 2. Beograd, 1961, pp. 1-48; Miroslav Pantić, "Jevreji...", op. cit., pp. 211-238; D. Nevecić-Grabovac, "Iz poezije...", op. cit., pp. 253-290; Vesna Mıović, Židovski.., op. cit., p. 27.

60 DAD, Diversa Notariae, XXVI., vol. CXVIII, fol. 112'.

61 Đ. KörBLER, "Život...", op. cit., p. 80; V. Mıovıć, Židovski..., op. cit., p. 28.

62 DAD, Cons. Min., vol. 51, fol. 15v; V. Mıović, Židovski..., op. cit., p. 28. 
ducati ungheresi alla sinagoga di Ragusa. ${ }^{63}$ Il suo testamento conferma la sua natura di scrittore-ponte capace di collegare diversi mondi. ${ }^{64}$

Didaco Pirro studiò in prestigiose scuole in Portogallo e a Salamanca in Spagna. Fece molti viaggi in Inghilterra, Belgio, Italia, Constantinopoli. Intorno al 1558 venne a Dubrovnik dove insegnava lingue classiche stringendo relazioni di amicizia con quasi tutti i nobili di riguardo del suo tempo: Dominik Zlatarić, Toma Budislavić, Miho Monaldi e altri. Nel 1545 fu consacrato alla fama internazionale grazie alla prima raccolta di poesie latine intitolata Carminum liber unus, in cui rivela una propensione verso motivi erotici e soluzioni fantasiose abbastanza pretenziose. Diplomato e letterato, fu onorato da Girolamo Faletti che lo incluse nei tredici brani della sua raccolta di poesie (1546). Giglio Giraldi lo prese nel suo trattato pionieristico sulla letteratura neolatina (1551). Alla sua patria adottiva Didaco espresse gratitudine in un'epistola scritta in esametri e dedicata a Paolo Manuzio, figlio del famoso editore Aldo: Ad Paulum, 1563. Con lo stesso spirito nel 1582 scrisse anche la poesia De illustribus familiis quae hodie Rhacusae exstant, meritoria del premio del Senato ragusino. ${ }^{65}$ Una piccola raccolta intitolata Encomiastes la dedicò all'amico e mecenate Toma Budislavić. Un'altra raccolta di tipo educativo-morale, secondo il titolo ma non sempre secondo il contenuto, è Cato Minor sive dysticha moralia ad Ludimagistros Olyssiponenses, 1592. Dalla raccolta di elegie in tre libri col titolo Elegiarum libri tres, dedicata a Dominik Zlatarić, fu stampata solo una parte. Il De D. Blasio, Rhacusanae Reipublicae Patrone, Carmen rimase un manoscritto. All'inizio del manoscritto si trova l'epistola: "Didaco Pirro, Portoghese saluta Domenico Zlatarich, rettore dell'istituto d'educazione padovano e cavaliere serenissimo". L'epistola introduttoria diretta a Dominik Zlatarić rivela un sincero rapporto tra i due, come anche il sincero attaccamento di Didaco alla sua patria adottiva ove trovò la pace e la possibilità di un'attività indisturbata. Questo scritto appartiene alle sue opere tardive. ${ }^{66}$ Il nostrano Zlatarić (1558-1613), un personaggio importante del circolo letterario raguseo della fine del Cinquecento e l'inizio del Seicento, traduttore dell'opera Aminta di Tasso, allora

63 DAD, Test. Not., 10.1., vol.51, fol. 27'-28'; Moises Orfalı, "Pogled na društveni i duhovni položaj sefardskih Židova u Dubrovniku na temelju zapisa javnih bilježnika (xvI. - xVII. stoljećr)", Dubrovnik 3 (2009), pp. 62-93.

64 Darko Novaković, "Didak Pir", Hrvatski biografski leksikon, vol. 3. Zagreb, 1993, pp. 372-374; Carlos Ascenso ANDRÉ, Um judeu no desterro: Diogo Pires e a memória de Portugal. Coimbra, Centro de Estudos Clássicos e Humanísticos da Universidade de Coimbra - I.N.I.C., 1992; António Manuel Lopes ANDRADE, O Cato Minor de Diogo Pires e a poesia didáctica do século xvı. Lisboa, Imprensa Nacional-Casa da Moeda, 2014. idem, "Os Senhores do Desterro de Portugal: judeus portugueses em Veneza e Ferrara em meados do séc. xVI", Veredas. Revista da Associação Internacional de Lusitanistas 6 (2006), pp. 65-108; idem, "A fábula na obra poética de Diogo Pires", Ảgora. Estudos Clássicos em Debate 9 (2007), pp. 99-118.

65 DAD, Cons. Rog. III., vol. LXVII., fol. 180: "Prima pars est de donando Doctori Didaco ob opusculum nobis dedicatum ducatos au. quindecim. - pXII v cXV".

66 Darko Novaković, Sveti Vlaho dubrovački parac u hrvatskoj književnosti, antologija; redazione: Luko Paljetak, Dunja FališEvc, Miljenko Foretić. Dubrovnik, Matica hrvatska Dubrovnik, 2001, pp. 25-31. 
studente a Padova, acquistò il manoscritto di Tasso pubblicandolo prima dell'autore stesso. ${ }^{67}$ L'amicizia di Didaco con il giovane Zlatarić, nata nello stesso anno di partenza del Lusitano da Dubrovnik, testimonia quanto questo intellettuale irrequieto fosse ben radicato nel nuovo ambiente. La poesia di Didaco è una fonte ricca di dati storico-culturali di Dubrovnik nella seconda metà del Cinquecento. Tra i ragusei che stringevano rapporti di amicizia con Didaco dobbiamo senz’altro segnalare Toma Budislavić (1545-1608), personaggio particolare, medico e vescovo, alunno delle Università di Bologna e Padova, che curava numerose persone notabili, come il sultano Muratto III. ${ }^{68}$ Incaricato dalla Santa Sede, si recò a Cracovia ove dimorò parecchi anni. Il vescovo di Cracovia Piotr Myszkowsky (1577-1591) lo nominò suo medico personale e poi canonico della diocesi. Nel circolo del vescovo Myyszkowski c'era anche Jan Kochanowski, uno dei più grandi poeti del rinascimento polacco. Due anni dopo il suo stanziamento in Polonia, furono conferite la dignità di nobile polacco e il titolo di medico regio a Tommaso Budislavić. Questo raguseo ebbe pure l'onore della dedica di un'opera del teologo polacco Stanislao Sokolowski. Il Budislavić sosteneva l'idea del panslavismo iniziata, a quanto pare, dal poeta di Dubrovnik Ilija Crijević-Cerva (1463-1529), seguito poi dal domenicano croato Vinko Pribojević. Durante il suo soggiorno a Cracovia Budislavić pubblicò una poesia in onore di Dubrovnik e della festività di S. Biagio, scritta da Didaco Pirro De illustribus... con la dedica all'autore. Lo stesso Didaco Pirro aveva pubblicato a Venezia prima della partenza di Budislavić in Polonia, un libretto con versi latini in onore di Dubrovnik. In Polonia a quel tempo la fama di Dubrovnik continuava a consolidarsi, grazie alla sua condizione di piccolo Stato cattolico e libero dal ruolo chiave nelle relazioni diplomatiche con l'Impero Ottomano. A questo scopo il Budislavić fece ristampare in sei varianti il libretto del suo amico Didaco Pirro, aggiungendovi alcune poesie proprie. $^{69}$

In ogni modo, Amatus Lusitanus e Didaco Pirro sono due personaggi interessanti della seconda metà del Cinquecento che hanno lasciato una traccia duratura nella storia culturale di Dubrovnik e nella cerchia umanistica ragusea.

67 Torquato TASSO, Ljuvene rane/Le piaghe d'amore, antologija hrvatskih prepjeva u povodu 400. obljetnice pjesnikove smrti, redazione Mirko Tomasović. Dubrovnik, Matica hrvatska, 1995, pp. 10-11.

68 Jorjo TADIć, Dubrovački portreti, vol. I. Beograd, Srpska književna zadruga, 1948, p. 355; Vinicije B. LuPIS, Dubrovnik i Poljska. Zagreb, Veleposlanstvo Republike Poljske u Zagrebu, 2005, p. 36.

69 Đ. Körbler, "Život...", op. cit., pp. 1-116; Anutn Kolendić, "Šest latinskih knjižica štampanih u Krakovu u čast Dubrovčanina Tome Natalisa Budislavića", Zbornik istorije književnosti Odelenja literature jezika Srpske akademije nauka i umetnosti, Lib. 3. Beograd, 1962, pp. 211-243, V. B. LuPIS, Dubrovnik..., op. cit., pp. 36-39; Š. LJUBIĆ, Dizionario..., op. cit., 1856, p. 255: "PIRRO FLAVIO GIACOMO (Didaco) ebreo, naque Evora nel Portogallo 1517. Espulso dalla patria, scorse l'Inghliterra, la Francia, il Belgo, la Svizzera, l'Italia, la Grecia, l'Egitto e buone parte dell'Asia, indi si ridusse in Ragusa, ove visse fino alla morte. Fu poeta latino tra primi del suo secolo, Una parte de'suoi carmi uscì alla luce nel 1852 presso Tommaso Natali in Cracovia, ristampati nel 1592 in Venezia sub signo leonis, e nel 1596 presso Felice Valriso. Le poesie inedite hanno un tal titolo: Didaci Pirrhi Lusitani elegiarum Libri III. ad D. Slatarichium Patav. Scholae rectorem et equitem splendissimum; accessit Lyricorum libellus eodem auctore." 


\section{BIBLIOGRAFIA}

\section{A. FONTES}

\section{Manoscritti e libri dello Didako Pir a Dubrovnik}

\subsection{Znanstvena knjižnica Dubrovnik:}

N. 217. "Jacobi Flavii Eborensis seu Didaci Pyrrhi Lusitani Carmina" "Elegia I Solimani Laudes" s napomenom pisanom olovkom "Didaci Pyrrhi stampata dell'Appendini”, "De illustribus familiis queae hodie Rhacusae extant, anno 1582. Cal. Ian. Ad amplissimum Senatum Rhacusanum (...)”, "De illustrissima urbe Rhacusa”.

N. 215. "Urbano Lampredio viro ubique gentium clarissimo Antonius Chersa Fratris sui Commentarium de vita et scriptis Flavii Iacobi Eborensis D.D.D. Elegia, Parve liber, prodi; jam prodi lucis in oras".

\subsection{Zavod za povijesne znanosti HAZU - Dubrovniku:}

N. D. a. 5. Commentarius Ludovici Cervarii Tuberonis de origine et incremento urbis Rhacusanae eiusdemque ditionis descriptio auctore Nicolao Joannis de Bona et Stephani Gradi antiquitatum Rhacusanarum brevis diatriba. His accedit de illustribus familiis, quae Rhacusae extant, ad amplissimum senatum elegia Didaci Pyrrhi. Cum notis et supplementis. Rhacusii 1790 typis Andrea Trevisan.

N. D. a 18, pag. 249 listova dall fine xvirI. secolo; 3 - 12 i 18 - 21: Jacobi Flavii Eborensis, / seu / Didaci Pyrrhi Lusitani / De Rhacusinae Urbis Laudibus / Carmen / quod habetur in Bibliotheca / Vaticana / inter Numero / 113333.

N. D. a. 29., "Ex Libris Antonii Luce de Sorgo": DIDACI / PYRRHI LVSITANI / ELEGIARVM LIBRI TRES / AD DOMINCVM SLATAEICCIVM PATA - / VINAE SCHOLAE RECTOREM ET EQVITAEM SPLENDIDISSIMVM / ACCESSIT LYRICORVM LIBELLVS EODEM AVCTORE: Elegia I. (“Ite procul...”), Elegia II (“Jam neque...”), Elegia III. (“Ergo ego...”), In Nuptiis Virginis Mandotiae et Petri Albani Elegia IIII., Ad Vincentium Porticum Rhacusanum Antistitem Elegia V., Elegia VI. (“Mamertina Chloe...”), Ad Nicolaum Gozzium Elegia VII., De origine domus Mensiae Elegia VIII., Elegia IX., (“Aegra iacet...”), Elegia X. Ad Simonem Benessium misit cum hor Hendecasyllabo, Hospes et Ianua Elegia CI. - Liber secundus. Ad Benessum. Elegia I., Elegia II. in obitum Mariae Gondulanae foeminae lectiss ad Georgium Gozzium illius uirum, De serrana Alpina. Elegia III. Ad Tassum poetam Tassi f. Elegia IIII., In qymnastica Mercurialis, Elegia V., Elegia VI. De origine Domus Saraccae apud Rhacusanos ad Joannem Saraccam equestris ordinis uirum, De commentarijs Indiae Medici 
Veronensis ad Dominicum Slatariccium iuuenem clariss. Et equestri dignitate insignem., Elegia VII., Elegia VIII., De ultimis Portugalliae Regibus qui aetate meae uixere, De Lusitanorum tumulo in urbe Ferraria. Elegia VIII., Momus ad Luccarum. Elegia X., Elegia XI. (“Quae belli...”) - Liber tertius. Elegia I. sub nomine Galli ueteris poetae edita, In obitum Joannae Etruriae Reginae, Elegia II., Elegia III. (“Non mihi smper...”), Michaeli Mensio Senatori grauiss et Aerarij IIII uiro S.D. - (In Mauri Vetrani Poetae Illyrici obitum. Elegia IIII., Elegia V. ad Proculianum astrologum, Ad Francinnum. Elegia VI. In obitum Nicaeae Paulii u. Manuccium Tuscar. Rer. Scriptorem, Ad Joannem Pinellium Ligurem Ode I., Ad Nicandrum Ode II., Ode III., (“Collige membra...”), Ad Lucium Lusitanum Ode IIII, In obitum Maurij poetae Illyrici scripsit postridie Kal. Feb. Ode V., De Urbe Rhacusa. Ode VI., Ad uirum clarissiumu Chysostomum Calabrum Antistitem Rhacusanum Ode VII. - lirica eiusdem Auctoris. Ad Aldumxoris Elegia VII., In Pulicem Elegia VIII., Renunciat amoribus Elegia IX., Elegia X. de exilio suo scripsit Nouae oppido Dalmatiae Hispanica clade nobilissimo. - Lirica eiusdem Auctoris, Ad Aldum Manuccium Tuscar. Rer. Scriptorem, Ad Joannem Pinellium Ligurem Ode I, Ad Nicandrum Ode II, Ode III (“Collige membra...”), Ad Lucium Lusitanum Ode IIII, In obitum Maurij poetae Ullyrici scripsit postridie Kal. Feb. Ode V. De Urbe Rhacusa. Ode VI., Ad uirum clarissimum Chrysostomum Calabrum Antistitem Rhacusanum Ode VII., Ad Giraldum senem Ode VIII., De nauicula Petri sub Gregorio XIII PM. Ode IX., De Tristano Cunio regis Portugalliae legato ad Leonem X. Pont. Max. Ode X., De Urbe Rhacusa pestilentia laborante, Ode XI. - Tabula omnium elegiarum et lyricorum libricorum libri secundam ordinem alphabeticum. Divus Blasius / Rhacusanus / Didaco Pyrrho Lusitano / Auctore, Didacus Pyrrhus Lusitanus Dominico Slatariccio Patavinae Scholae Rectori, et Equiti splendissimo S. D. - proza, Ad Divium Blasium, D. Blasius. 


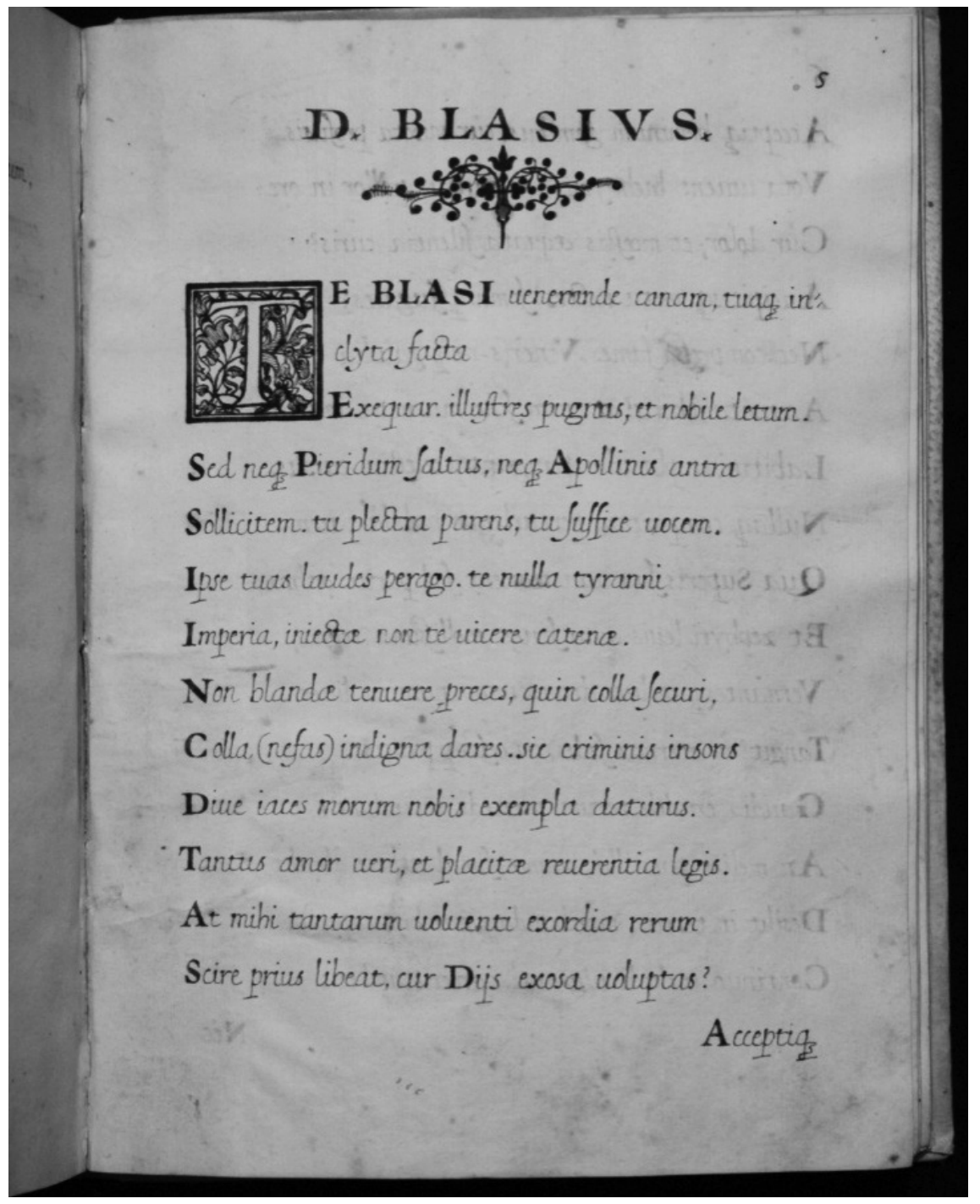

N. D.a. 40. Marko Bruerović, Volgarizzamento di varie poesie latine - Džono Restić, Carmina): Variorum auctorum / Latina Carmina / Italicis versibus reddita / à Marco Bruerio / Cive Gallo. Ili: Volgarizzamento / di varie Poesie Latine / d'Autori diversi / tradotte in verso Volgare / da Marco Bruere / Cittadino Francese.

\subsection{Franjevački samostan Male braće- Dubrovnik}

N.162. Antun Kerša/Chersa (1779. - 1838.), "Urbano Lampredi Anti. Chersa fratris sui Commentarium de vita et scriptis Flavii Jacobi Eborensis... elegia”. 


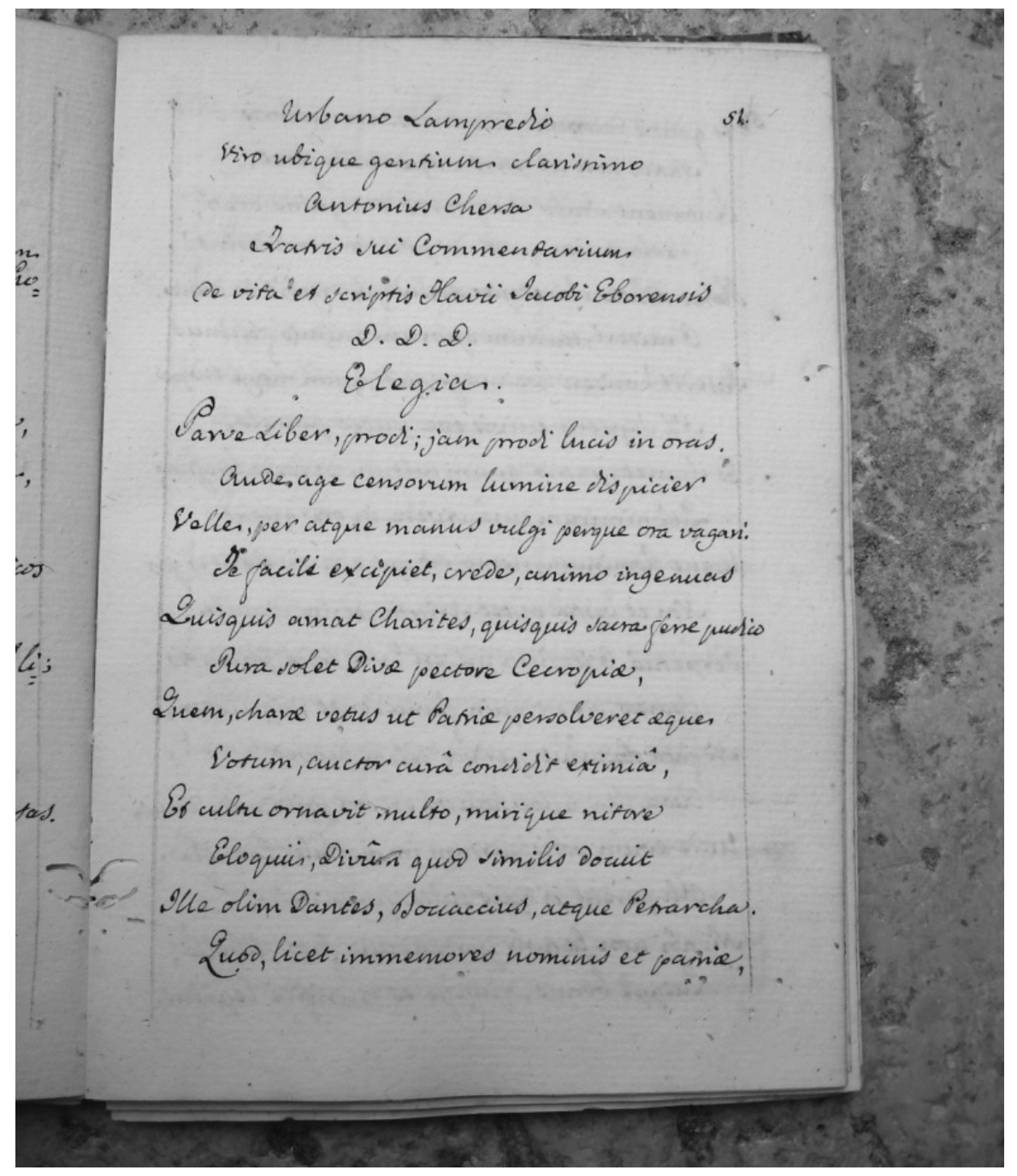

N. 228. "Flavii Jacobi Eborensis ac Didaci Pyrrhi Carmina, MS Aloysii Garmogljesi. Rhacusae Anno Domini 1806”, Scripsi die 19. decembris 1806.”: "Flaviii Jacobi Eborensis Cato Minor, sive Disticha Moralia ad ludimagistros Olysipponenses. Accessere Epigramata, et moralia noñulla eodem auctore, quae sequens pagella indicabit. Opus pium, ac erudiendis pueris apprime necessarium. Venite filii, audite me, timore Domini docebo vos, Osal. 33". Flavius J. Eborensis olysipponensibus ludimagister. S.D. Flavius Jacobus Joanni typographo S.D.

Ad Vicentium Gilianum, In obitum Francisci Medicis Magni Etrurie Ducis, Lactantii monaci Cassinati ad christianum puerum pia exhortio, Acia Monti theologi Hispalensis, Theopili Allobrogis apud Bituricenses ludimagistri, Henrici Angli Jesuitae e Seminario Augustano Gulielmi Budaei adolescentis, Antonii Fulgosii 
Liguris, Dionisyii Hedui D. Germani Abbatis, Valdaurae Medici Valentini in Jacobi Xenia, Martini Gamboae theologi Cantabri carmen.

Praefatio in I. lib. Moralium Distichorum. Ad Antoinum Medicen Capellum Francisci Magni Etruriae D. F. Ad Lectore,

Moralium Distichorum lib. I.

Moralium Distichorum lib. II. Praefatio in II. libr. Ad Ant. Medicen Franc. Magni Etruriae Ducis F. Ad lectorem. Moralium distichorum lib. II.

Moralium Distichorum lib. III. Ad Marinum Bonum patricium ragusinam et senatorem petit sibi commodori Secundi Hagiensis carmina.

Moralium Carminum lib. I. Ad Antonium Medum S.D. Ad eumedum, Fugienda vita et amplectenda virtus, Ad Marcellum de vita brevitate, Qui vere vitalem vitam agit, Vera felicitas in quo consistat, Et erit sicut arbor plantata etc. Ex. I. Ps., Fortunae volubilitas, De Nicolao modestissimo adolescente primam missam celebrante i D. Blasiii aede, Temporis jactura maxima ex Cuspiniano avaro hypocrita, Boni judicis officum, De Regno et publica, Qui vere felix, Unusquisque fortunae suae faber, Epigrammatum libellus ad Ioannem Darsam, De sacello Virg. Rosarii, quod Ragusae constructum est an. 1594., De urbe Rhacusa ab egregio pictore expressa, Ad Matthaeum Benessam Rhacusanum rectorem, Ad eundem, Gratulatur Dominico Slatarichio nobili juveni et Patavinae inscribendum.

Hendecasyllabi: In Gallum poetam maledicum, Ad Thomam, De Portico Rhacusano epo, Vinosii hendecasyllabi ad Matthaeum Benessam V.C., Ad Battidurum, Ad Musorium, Ad Matthaeum Benessam, Ad Simonem Benessam vindemiantem, Ad Aldum Manucium Tuscarum rerum scriptorem.

Lyrica: Ode I. In obitum Mauri poetae Illyrici, ode II. De Urbe Rhacusa, Ode III. De eadem Urbe pestilentia laborante ad Hieronymum Cervinum (...), Ode IV. Ad Michaelem Bonum.

Tumuli: Ad lectorem, Ad P. Maurum Melitensem Abbatem, Dominici Cervii epitaphium, simonis Aurii Michaelis Fili et Catharinae matris Epitaphium (...), Gregori Natalis OP epitaphium, Fr. Bonifatii epitaphium, Mauri Vetranii epitaphium, Marci Rhanniae rag. Archidiaconi epitaphium, Aliud in eundem.

De illustribus familiis: De illustribus familias quae hodie rhacusae extant an. MDVC. Cal. Jan., Due epigrammata Dominico Slatarichio.

Elegiarum lib. III.

Elegiarum lib. I. Elegia I., El II., El. III., Elegia IV., In nauptiis virginis Mandotiae et Petri Albani, Elegia V. Ad Vincentium porticum rag. Antistitem, Elegia VI., Elegia VII. Ad Nicolum Gozzium, Elegia VIII. De origine domus Mensiae, Epigramma, Elegia IX., Elegia X. quam poeta misit ad Simonem Benessam cum hoc hendecasyllabi. Ad eundem epigramma, Elegia XI. Dialogismus inter hospitem et januam.

Lib. II. Eleg. I. Ad Benessum, Elegia II. In obitum Mariae Gundulanae ad Gregorium Gozzium illius virum, Elegia III. de Serrana Alpina, Eleg. IV. Ad Tassum poetam Tassi filium, El. V. In gymnastica mercurialis, Elegia VI. Ad Joannem Saraccam de origine domus Saraccae apud Rhacusanos, El. VII. Ad Dom. Slatarichium de Commentariis Indiae Medici Veronensis.

De ultimis Portugalliae regibus qui aetate mea vixere argumentum 
Eleg. VIII., Eleg. IX. De Lusitanorum tumulo in urbe Ferraria, El. X. momus ad luccarum, El. XI. Epigramma ad Dominicum Rhaniam.

Lib. III. Eleg. I. Sub nomine Galli veteris poetae edita, El. II. In obitum Joannae Etruriae reg., Epigramma de duabus sororibus, Ambrosio Morali Cordubensi S. D., El. III., El. IV. In Mauri Vetrani poetae illyrici obitum, El. V. Ad Proculianum astrologum, El. VI. Ad Francinnum, El. VII. In obitum Nicolae Pauli uxoris, El. VIII. In Vulicem, El. IX. Renunciat amoribus, El. X. De exilio suo. Scripsit Novae oppido Dalmatiae hispanica clade nobilis.

Lyrica: Ode I. Ad Ioannem Pinellium Ligurem, ode II. Ad Nicandrum, Ode III., Ode IV. Ad Lucium Lusitanum, Ode V. Ad Giraldum Senem, Ode VI. De navicula petri sub Gregorio XIII., Ode VII. De Tristano Cunio regis Portugalliae legato ad Leonem X.

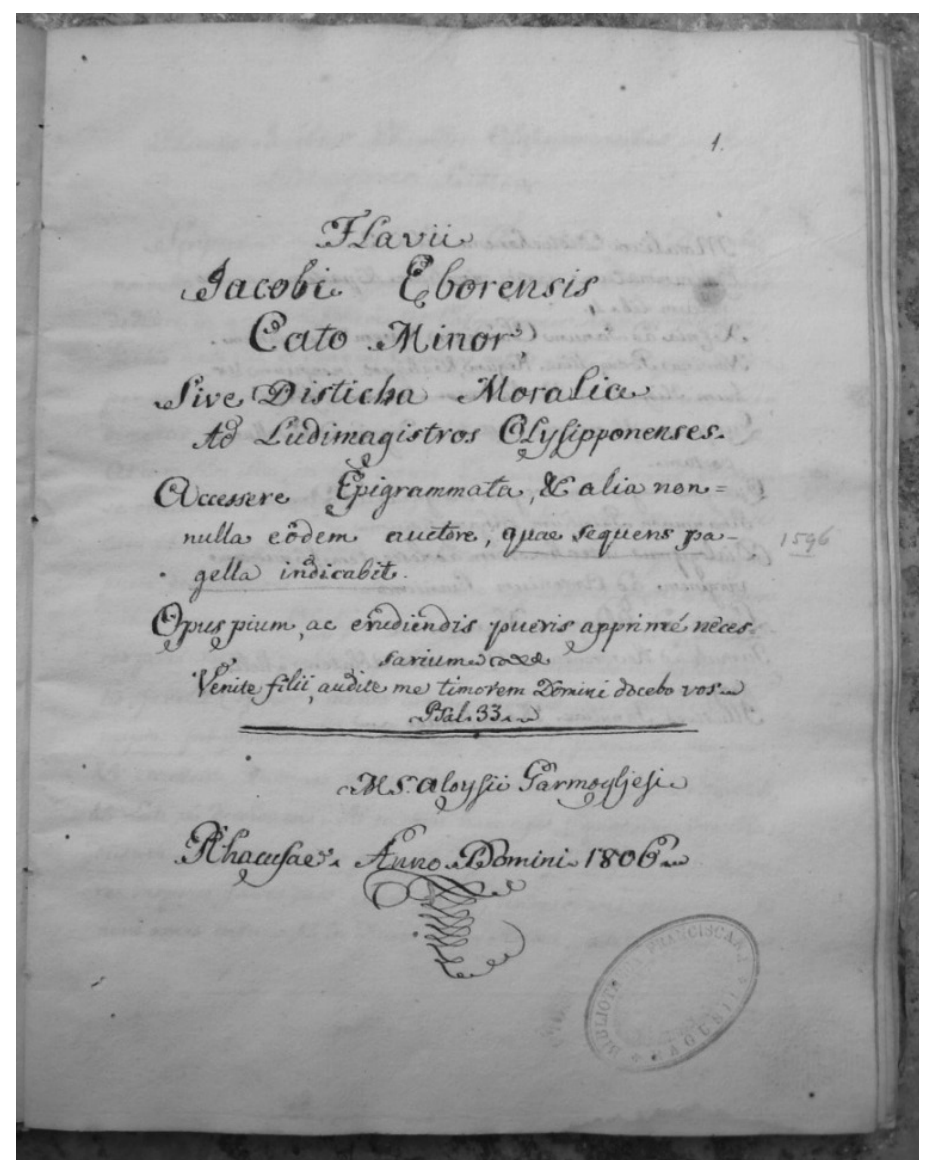

NN. 453., Zibaldone III, Memorie storiche su Ragusa raccolte dal P. Gian Maria Mattei. 


\subsection{Državni arhiv a Dubrovnik}

DAD. XXI. 2. Memoriae - Cronache - Diarii - Manuscripta diversa - Curiosa, N. 42: Jacobi Flavii Eborensis, seu Didaci Pyrrhi De Ragusinae Urbis Laudibus Carmen, Inscriptum habetur in Bibliotheca Vaticana/ inter miscellanea typis vulgatorum/ Volumine quod insignitur numero 11333./ Ferrariae/ Apud Valentem Panizzam Mantuananum/ Ducalem Typographium/ 1563.

DAD. XXI. 2. Memoriae - Cronache - Diarii - Manuscripta diversa - Curiosa, N. 69. De Didache Pirro, Appunti, estratte ex.

DAD. XXI. 2. Memoriae - Cronache - Diarii - Manuscripta diversa - Curiosa, N. 95. T. Chersae de Didaci Pirri Commentario: (...)Hic Accedit de Illustribus Familis, quae Rhacusae Extant ad Amplissimum Senatum Elegia Didaci Pyrrhi. Cum notis Suplementis(...).

R 742 copia dalla biblioteca Raczyńskich da Poznanj, copiata da Władisław Gluck: ENCOMIASTES / CARMINE ELEGIACO / AD RNDUM D. THOMAM NATALEM / Rhacusianum, Art. \& Medic. Doctorem / praestantissimum, necon Ca- / nonicum Cracuien. / dignissimum. / CRACOVIAE / In officina Lazari. Anno Domini / M.D. LXXXIII.

R 265b DELLA / VITA E DEGLI SCRITTI / DI / DIDACO PIRRO / ALTRAMENTI DETTO / IACOPO FLAVIO EBORENSE; / COMENTARIO / DI / TOMMASO CHERSA / FIRENZE / NELLA STAMPERIA MAGHERI / 1826.

R 350 COMMENTARIOLUS / LUDOVICI CERVARII TUBERONIS / De Origine \& incremento Urbis Rhacusanae / EJUSDEMQUE DITIONIS DESCRIPTIO / AUCTORE / NICOLAO JOANNIS DE BONA, / ET / STEPHANI GRADI / ANTIQUITATUM RHACUSANARUM BREVIS / DIATRIBA / His accedit de illustribus Familiiis, quae Rhacusae extant / ad amplissimum Senatum / ELEGIA DIDACI PYRRHI / Cum notis \& Supplementis. / THACUSII MDCCXC. / TYPIS ANDREAE TREVISAN. Indietro e elegia: DE ILLUSTRIBUS FAMILIIS, QUAE RHACUSA/ EXTANT AD AMPLISSIMUM SENATUM DIDACI PYRRHI/ ELEGIA.

\section{B. BIBLIOGRAFIA:}

Amati Lvsitani, Curationum medicinalium, Centuriae duae, Quinta et Sexta (...). Lugduni, apud Gulielmum Rouillium, 1564.

Andrade, António Manuel Lopes, O Cato Minor de Diogo Pires e a poesia didáctica do século Xvi. Lisboa, Imprensa Nacional-Casa da Moeda, 2014.

Andrade, António Manuel Lopes, "Os Senhores do Desterro de Portugal: judeus portugueses em Veneza e Ferrara em meados do séc. xvi”, Veredas. Revista da Associação Internacional de Lusitanistas 6 (2006), pp. 65-108. 
Andrade, António Manuel Lopes, "A fábula na obra poética de Diogo Pires", Ágora. Estudos Clássicos em Debate 9 (2007), pp. 99-118.

Andrade, António Manuel Lopes; Crespo, Hugo Miguel, "Os inventários dos bens de Amato Lusitano, Francisco Barbosa e Joseph Molcho, em Ancona, na fuga à Inqusição (1555)”, Ágora. Estudos Clássicos em Debate 14.1 (2012), pp. 45-90.

André, Carlos Ascenso, Um judeu no desterro: Diogo Pires e a memória de Portugal. Coimbra, Centro de Estudos Clássicos e Humanísticos da Universidade de Coimbra - I.N.I.C., 1992.

Anselmi, Sergio, "Motivazioni economiche della neutralità di Ragusa nel cinquecento" in Antonio Di VIttorio, Sergio Anselmi, Paola Pierucci, Ragusa (Dubrovnik) una repubblica adriatica. Saggi di storia economica e finanziaria. Bologna, Cisalpino, 1994, pp. 25-34.

Appendini, Francesco Maria Notizie istorico-critiche sulle antichità, storia e letteratura de'Ragusei, vol. II. Ragusa, dalle stampe di Antonio Martecchini, 1803.

Braudel, Ferdinand, Civiltà e Imperi del Mediterraneo nell'età di Filippo II. Torino, Einaudi, 1982

Brlek, Mijo, “Lodovico Beccadelli”, Hrvatski biografski leksikon, sv. I. Zagreb, 1983, pp. 562 - 565.

Bülent, Ari, "1. Appendix to the text "An Influental Jew on the Ottoman Court: David Pass": in Mirjana Polić Bobić (ed.), Tajna diplomacija u Dubrovniku u xvi. Stoljeću. Zagreb, Sveučilište u Zagrebu, 2011, pp. 194-198.

Carter, F. W., Dubrovnik (Ragusa). A Classic City-State. London-New York, Seminar Press, 1972.

DugačKi, Vladimir, “Amatus Lusitanus", Hrvatski biografski leksikon, vol. I. Zagreb, 1983, p. 237.

Efron, Z., "Jevrejski nadgrobni spomenici u Dubrovniku”, in Zbornik Židovskog muzeja 1 (1971), pp. 337-340.

Far lati, Daniele, Illyricum sacrum, vol. VI. Venezia, 1800.

Freiberger, Šalom, “Povijest Židova u Dubrovniku do izgona god. 1515.”, Omanut 1 (1936), pp. 30-37.

Ginsberg, David, "Mletački rabin Leo de Modena i dubrovački pjesnik Vinko Komnenović", Omanut, 10 (1940), pp. 156-161.

Glesinger, Lavoslav,“Dvije studije o Amatusu Lusitanusu”, Židov, vol. I, 20 (1937), p. 83.

Glesinger, Lavoslav, Amatus Lusitanus i njegov lječnički rad u Dubrovniku (1556-1558). Zagreb-Beograd, Biblioteka Jevrejskog narodnog kalendara, 1940.

Glesinger, Lavoslav, "Amatus Lusitanus i njegov liječnički rad u Dubrovniku (1556-1558)”, Omanut 3-4 (1940), pp. 61-62.

Glesinger, Lavoslav, "Dubrovački lječnik - prvi borac protiv progona vještica”, Zaštita zdravlja 3 (1948), pp. 106-107.

GLEsINGER, Lavoslav, “Dubrovački lječnik Amatus Lusitanus”, Zbornik Židovskog muzeja 1 (1971), pp. 291-312. Gučetıć, Nikola Vitov, Dialogo della Bellezza/Dijalog o ljepoti i Dialogo d’amore/Dijalog o ljubavi. Zagreb, Matica hrvatska, 2008.

Gušić, Mirjana, "Neki primjerci tekstila u zbirci sinagoge jevrejske općine u Dubrovniku”, Zbornik Židovskog muzeja 1 (1971), pp. 325-336.

Harris, Robin, Povijest Dubrovnika. Zagreb, Golden marketing-Tehnič ka knjiga, 2006. 
Hrabak, Bogumil, “Jevreji u Albaniji od kraja XIII do kraja Xvir veka i njihove veze s Dubrovnikom”, Zbornik Židovskog muzeja 1 (1971), pp. 55-97.

Jeremić, Risto; Tadić, Jorjo, Prilozi za istoriju zdrastvene kulture starog Dubrovnika, vol. 2. Beograd, 1939.

KeršA, Toma, Della vita e degli scritti di Didaco Pirro altrimenti detto Iacopo Flavio Eborense. Commentario di Tommmaso Chersa. Firenze, nella stamperia Magheri, 1826.

Kolendić, Petar, "Nekoliko pesama humaniste Didaka Pira”, Zbornik istorije književnosti Odeljenja literature i jezika Srpske akademije nauka i umetnosti, lib. 2. Beograd, 1961, pp. 1-48.

Kolendić, Anutn, "Šest latinskih knjižica štampanih u Krakovu u čast Dubrovčanina Tome Natalisa Budislavića”, Zbornik istorije književnosti Odelenja literature jezika Srpske akademije nauka i umetnosti, lib. 3. Beograd, 1962, pp. 211-243.

Körbler, Đuro, “Život i rad humanista Didaka Pira Portugalca, napose u Dubrovniku”, RAD JAZU, lib. 216 (1917), pp. 1-169.

Krasić, Stjepan; Razzi, Serafino, Povijest Dubrovačke metropolije i dubrovačkih nadbiskupa (X. - XVI. stoljeća). Dubrovnik, Biskupski ordinarijat, 1999.

KReKIĆ, Bariša, "The role of the Jews in Dubrovnik (thirteenth-sixteenth centuries)", Viator (Medieval and Renaissance Studies) 4 (1973), pp. 257-272.

Krekić, Bariša,"The role of the Jews in Dubrovnik (thirtheen-sixteenth centuries)", in Bariša Krekić, Dubrovnik, Italy and the Balkans in the Late Middle Ages. London, Variorum Reprints, 1980, XXI, pp. 257-272.

Krekić, Bariša, Dubrovnik: a Mediterranean Urban Society, 1300-1600. Great Yarmouth, 1997.

Ljubić, Šime, Dizionario biografico degli uomini illustri della Dalmazia. Beč, 1856.

Lupis, Vinicije B., Dubrovnik i Poljska. Zagreb, Veleposlanstvo Republike Poljske u Zagrebu, 2005.

Miović, Vesna, Židovski geto u Dubrovačkoj Republici (1546-1808). Dubrovnik-Zagreb, HAZU, Zavod za Povijesne Znanosti u Dubrovniku, 2005.

Monster, Ladislav, "Proces zbog "ritualnog ubistva" u Dubrovniku 1502 godine i tragična smrt lekara Moše Maralija”, Zbornik Židovskog muzeja 1 (1971), pp. 99-112.

Mortidjija, Tias, “Židovi u starom Dubrovniku”, Hrvatska straža 6 (1934), pp. 221-225.

Nevenić-Grabovac, D., "Iz poezije Didaka Pira”, Zbornik Židovskog muzeja 1 (1971), pp. 253-290.

Novaković, Darko, “Didak Pir”, Hrvatski biografski leksikon, vol. 3. Zagreb, 1993, pp. 372-374.

Novaković, Darko, Sveti Vlaho dubrovački parac u hrvatskoj književnosti, antologija; redazione: Luko Paljetak, Dunja Fališevc, Miljenko Foretić. Dubrovnik, Matica hrvatska Dubrovnik, 2001.

Pantić, Miroslav “Jevreji u dubrovačkoj književnosti”, Zbornik Židovskog muzeja 1 (1971), pp. 211-238.

Pasarić, Antun, "Pjesme Didaka Pira”, Srd, Dubrovnik, 1903, pp. 91-92, p. 141, pp. 184-186, pp. 215-218.

Pires, Diogo, Cato Minor siue Disticha Moralia ad Ludimagistros Olysipponenses (...). Venetiis, apud Felicem Valgrisium, 1596.

Polıć Boвıć, Mirjana, Među križem i polumjesecom, dubrovačke dojave španjolskom dvoru o Turcima u Xvi. Stoljeću. Zagreb, Naklada Ljevak, 2000. 
Polić Bobić, Mirjana, "Activities of the Spanish Secret Service in the Republic of Dubrovnik in the $16^{\text {th }}$ Century and the Role of Spanish Confidant Marin Zamanja”, in Mirjana Polıć Bobić (ed.), Tajna diplomacija u Dubrovniku u xvi. Stoljeću. Zagreb, Sveučilište u Zagrebu, 2011, pp. 181-190.

Polıć Bobıć, Mirjana, “Španjolska tajna služba u Dubrovačkoj Republici u xvi. stoljeću i uloga španjolskog povjerenika Marina Zamanje u njoj”, in Mirjana Polić BobIć (ed.), Tajna diplomacija u Dubrovniku u xvi. Stoljeću. Zagreb, Sveučilište u Zagrebu, 2011, pp. 86-93.

Popović, T., "Dubrovnik i Ankona u jevrejskoj trgovini xvi veka”, Zbornik Židovskog muzeja 1 (1971), pp. 41-53.

ReŠEtar, Milan, “Amat Luzitanac, dubrovački ljekar xvi. Vijeka”, Brankovo kolo 6 (1900), pp. 39-41.

TADIĆ, Jorjo, Jevreji u Dubrovniku do polovine Xvir. Stoljeća. Sarajevo, Izdala La Benevolencia, 1937.

TADIĆ, Jorjo, Dubrovački portreti, vol. I. Beograd, Srpska književna zadruga, 1948.

TAsso, Torquato, Ljuvene rane/Le piaghe d'amore, antologija hrvatskih prepjeva u povodu 400. obljetnice pjesnikove smrti, redazione Mirko Tomasović. Dubrovnik, Matica hrvatska, 1995.

Torbarina, Josip, "Fragmenti iz neizdanih pisama Lodovika Beccadellija 1555-1564”, Dubrovačka, mjesečna ilustrovana revija I (1929), pp. 9-10.

SAmardžIČ, Radovan, “Dubrovački Jevreji u trgovini xvi i xvıI veka”, Zbornik Židovskog muzeja 1 (1971), pp. 21-39.

Šı, Lavoslav, Die jüdische Ärtze in Jugoslavien. Osijek, E. Sekler, 1931.

ŠUndrica, Zdravko, "Popis stanovništva Dubrovačke republike iz 1673-74 godine”, Arhivski vjesnik 2 (1959), pp. 419-456.

Švelec, Franjo, “Marin Držić”, Hrvatski biografski leksikon, vol. III. Zagreb, 1993, pp. 631-636.

Švelec, Franjo, "Vlaho Držić", Hrvatski biografski leksikon, vol. III. Zagreb, 1993, pp. 636-637.

Vekarič, Nenad, Vlastela grada Dubrovnika, 2. Vlasteoski rodovi (A-L). Zagreb-Dubrovnik, Zavod za povijesne znanosti HAZU u Dubrovniku, 2012.

Vekarič, Nenad, Vlastela grada Dubrovnika, 3. Vlasteoski rodovi (M-Z). Zagreb-Dubrovnik, Zavod za povijesne znanosti HAZU u Dubrovniku, 2012.

VekArIČ, Nenad, Vlastela grada Dubrovnika, 4. Odabrane biografije (A-D). Zagreb-Dubrovnik, Zavod za povijesne znanosti HAZU u Dubrovniku, 2013. 
A partir dos alvores do século XVI, a matéria médica torna-se indiscutivelmente um tema de primeira grandeza entre os membros da República das Letras, objecto de estudo e de controvérsia entre os mais notáveis humanistas europeus, em particular entre os cultores da arte médica. Entre os autores em destaque neste volume encontram-se, à cabeça, os nomes de Amato Lusitano, Garcia de Orta e Nicolás Monardes, famosos pelos contributos valiosos que deram para o conhecimento do mundo natural. 0 volume encontra-se dividido em duas partes: a primeira, subordinada ao título "Humanismo e Ciência", alberga os estudos que versam sobre todos os autores estudados, à excepção de Amato Lusitano; a segunda está reservada a um conjunto de trabalhos dedicados exclusivamente ao médico albicastrense, cuja autoria se fica a dever, em boa parte, aos membros da equipa do projecto de I\&D "Dioscórides e o Humanismo Português: os Comentários de Amato Lusitano", tomando, por isso, 0 seu próprio título. Nesta segunda parte, oferece-se, desde já, aos leitores uma amostra significativa do trabalho desenvolvido no âmbito do projecto e que culminará, assim se espera, na edição e tradução integral para língua portuguesa das quatro obras previstas de Dioscórides, Amato Lusitano e Pietro Andrea Mattioli. 
